\title{
Partition functions, loop measure, and versions of SLE
}

\author{
Gregory F. Lawler* \\ Department of Mathematics \\ University of Chicago \\ 5734 University Avenue \\ Chicago, IL 60637-1546 \\ lawler@math.uchicago.edu
}

February 10, 2009

\begin{abstract}
We discuss the partition function view of the Schramm-Loewner evolution. After reviewing a number of known results in the framework of Brownian loop measures and scaling rules for partition functions, we give some speculation about multiply connected domains.
\end{abstract}

\section{Introduction}

The Schramm-Loewner evolution (SLE) has become an invaluable tool in studying scaling limits of critical two-dimensional lattice models in statistical mechanics. We will not describe $S L E$ in detail here, allowing the reader to consult [9, 4, 6, 10], e.g., but will start with some general remarks.

Many lattice models in statistical physics (self-avoiding walk, loop-erased walk, percolation, Ising model,...) are defined on a lattice. If one considers a finite piece of the lattice and gives appropriate boundary conditions, then the models are elementary, giving certain weights to walks or configurations. In the models we consider, these weights depend on a parameter and for a certain critical value of the parameter one hopes to get a scaling limit. In two dimensions, as originally predicted in $[1,2]$, the scaling limit is often conformally invariant. The scaling limit is a measure on some kind of configuration in the continuum limit; the examples we consider here are all measures on curves. In taking the scaling limit, one often has to normalize the measure; at criticality, this normalization is typically of the

${ }^{*}$ Research supported by National Science Foundation grant DMS-0734151. 
order of a power of the lattice spacing in contrast to an exponential correction off of criticality. This normalization leads to scaling behavior of the limit object which is conformally "covariant" rather than invariant.

Oded Schramm [9] made the major breakthrough in this area when he considered two models, loop-erased random walk and the percolation exploration process (boundary of percolation clusters with a particular boundary condition) which were conjectured to give probability measures in the scaling limit. His beautiful argument showed that if one had a probability measure on curves from 0 to $\infty$ in the upper half plane that satisfied conformal invariance and another property that is called the domain Markov property, then the curves must be in a one-parameter family of curves called chordal $S L E_{\kappa}$. He gave a similar result for curves from a boundary to an interior point giving radial $S L E_{\kappa}$. Starting from radial $S L E_{\kappa}$ one can also construct a measure on curves from 0 to $\infty$ in $\mathbb{C}$, and this is called whole plane $S L E_{\kappa}$. One of Schramm's starting assumptions was that $S L E_{\kappa}$ produced a conformally invariant family of measures. For this reason, it was natural to define $S L E_{\kappa}$ on other domains by conformal invariance.

However, one should also be able to define $S L E_{\kappa}$ on other domains as scaling limits. Understanding this led to development in the theory of $S L E$. For example, the realization that self-avoiding walks satisfy a "restriction property" led to asking which $S L E_{\kappa}$ have this property [7]. For other values of $\kappa$ a similar relationship was found but there was a correction term that can be interpreted in terms of Brownian loops. Also in [7], the $S L E(\kappa, \rho)$ processes were defined which are one way to describe $S L E$ paths that are locally absolutely continuous with respect to chordal $S L E_{\kappa}$.

Another approach, and the one emphasized in this paper, is in terms of (normalized) partition functions. One can understand $S L E_{\kappa}$ in different domains by considering the Radon-Nikodym of the measure on one domain with respect to the other. The evolution of this over time generally leads to a (local) martingale. This approach was emphasized in [6] and examples are given there describing the relationship between the original lattice partition functions and the normalized partition functions in the limit. If one knows the partition function, one can obtain conditional distributions by means of the local martingale and the Girsanov theorem.

Section 2 describes the partition function view of $S L E$ measures as seen through measures that are absolutely continuous with respect to chordal $S L E_{\kappa}$. All of the examples in this section have appeared before although there is some novelty in the presentation here. A reason to include it here is to emphasize that there are essentially two terms in the RadonNikodym derivatives for paths stopped at a stopping time: a Brownian loop term which involves the interaction of the curve with the boundary and a "partition function" term which describes the measure of the set of possible ways of extending the path. This gets a little more complicated when considering multiple paths so in Section 2.6 a measure is defined that only considers the first (loop) term and not the continuation term.

Section 3 discusses a number of natural ways to extend definitions using a partition function and Brownian loop measure perspective. We discuss one way (there is not a unique way) to extend $S L E$ to multiply connected domains. In the particular case of the annulus, we 
conjecture that this is the correct way to describe radial $S L E_{\kappa}$ from an interior to a boundary point. A basic theme is that there are two base measures: $S L E$ starting at a boundary point (chordal) and SLE starting at an interior point (whole plane) and one should be able to define other versions using Brownian loops and appropriate partition functions.

The Brownian loop measure is an important part of this discussion, and for this reason we give a discussion of it in the final section. The emphasis here is on how to compute or estimate measures of sets of loops.

I have not listed many references in this paper. This is not to imply that there have not been many contributors to $S L E$; in fact, many of the ideas in the next section have been discussed by many authors. There is an extensive bibliography on $S L E$ in my notes [6].

\section{Partition functions and $S L E$}

Throughout this section we assume $0<\kappa \leq 4$ unless we explicitly state otherwise.

\subsection{Chordal $S L E$ as a probability measure}

We will give a quick review of the definition of chordal $S L E$ in simply connected domains, see $[4,6,10]$ for more details. Chordal $S L E_{\kappa}$, as defined by Schramm, is a family of probability measures $\mu_{D}^{\#}(z, w)$ indexed by simply connected proper subdomains $D$ of $\mathbb{C}$ and distinct boundary points $z, w$. These measures are supported on noncrossing curves $\gamma:\left(0, t_{\gamma}\right) \rightarrow \bar{D}$ with $\gamma(0+)=z, \gamma\left(t_{\gamma}-\right)=w$; more precisely, the measures are supported on equivalence classes of curves where two curves are equivalent if one is an increasing reparametrization of the other. The family satisfies the following two assumptions.

- Conformal invariance. If $f: D \rightarrow f(D)$ is a conformal transformation, then

$$
f \circ \mu_{D}^{\#}(z, w)=\mu_{f(D)}^{\#}(f(z), f(w)) .
$$

(The notation $f \circ \mu_{D}^{\#}(z, w)$ is shorthand for the measure on curves $\gamma$ in $f(D)$ obtained by taking the measure of $f^{-1} \circ \gamma$.)

- Domain Markov property. Suppose an initial segment $\gamma[0, s]$ of the curve is observed. Then (with respect to the probability measure $\left.\mu_{D}^{\#}(z, w)\right)$ the conditional distribution of $\gamma\left[s, t_{\gamma}\right]$ given $\gamma[0, s]$ is given by

$$
\mu_{D \backslash \gamma[0, s]}^{\#}(\gamma(s), w) \text {. }
$$

By the Riemann mapping theorem, it suffices for defining such a family to give $\mu_{\mathbb{H}}^{\#}(0, \infty)$ where $\mathbb{H}$ denotes the upper half plane. If we choose a convenient parametrization of $\gamma$ by half-plane capacity and let $g_{t}$ denote the conformal transformation of $\mathbb{H} \backslash \gamma(0, t]$ onto $\mathbb{H}$ 
satisfying $g_{t}(z)-z=o(1)$ as $z \rightarrow \infty$, then $g_{t}$ satisfies the (chordal) Loewner differential equation

$$
\partial_{t} g_{t}(z)=\frac{2}{g_{t}(z)-V_{t}}, \quad g_{0}(z)=z .
$$

This equation is valid for any noncrossing continuous curve $\gamma$, but the assumptions on the family $\mu_{D}^{\#}(z, w)$ above imply that $V_{t}$ must be a driftless one-dimensional Brownian motion. Chordal $S L E_{\kappa}$ is obtained by letting $V_{t}=g_{t}(\gamma(t))=U_{\kappa t}$ where $U_{t}$ is a standard Brownian motion. The measure $\mu_{D}^{\#}(z, w)$ for other simply connected $D$ is defined by conformal transformation.

If we make a linear change of time, we can write

$$
\partial_{t} g_{t}(z)=\frac{a}{g_{t}(z)-U_{t}}, \quad g_{0}(z)=z,
$$

where $a=2 / \kappa$ and $U_{t}=g_{t}(\gamma(t))$ is a standard Brownian motion. This makes some formulas slightly nicer and we will use this parametrization; one only need remember that if $g_{t}$ satisfies (2), then $g_{t}^{*}=g_{\kappa t}$ satisfies (1).

For $0<\kappa \leq 4$, the measure $\mu_{\mathbb{H}}^{\#}(0, \infty)$ is supported on simple (non-self-intersecting) curves; for $\kappa>4$, the curves have self-intersections. For $\kappa \geq 8$, the paths are plane-filling. Three important parameters associated to the measure are the boundary scaling exponent

$$
b=b_{\kappa}=\frac{6-\kappa}{2 \kappa}
$$

the (one-sided) interior scaling exponent

$$
\tilde{b}=\tilde{b}_{\kappa}=\frac{\kappa-2}{4} b=\frac{(6-\kappa)(\kappa-2)}{8 \kappa},
$$

and the central charge

$$
\mathbf{c}=\mathbf{c}_{\kappa}=(3 \kappa-8) b=\frac{(3 \kappa-8)(6-\kappa)}{2 \kappa} .
$$

We will write quantities in terms of $b, \tilde{b}, \mathbf{c}$; this gives implicit dependence on $\kappa$.

If $T$ is a stopping time for the Brownian motion, we can consider $S L E_{\kappa}$ as a probability measure on paths $\gamma(0, T]$. We will write $\mu_{\mathbb{H}}^{\#}(0, \infty)$ for this probability measure as well leaving implicit the dependence on $T$. Stopping times $T$ can be thought of as stopping times for the path $\gamma$; to determine whether or not to stop at a time $t$ one can only look at $\gamma(0, t]$.

The measure on paths $\gamma(0, T]$ is a probability measure on paths starting at $\mathbb{H}$, stopped at time $T$, but known to continue eventually to $\infty$. We state this to emphasize that the curve $\gamma$ near time $T$ does not look like the reversal of a one-sided path starting at $\gamma(T)$. This latter measure is described by versions of whole plane $S L E$ that we discuss later in this paper.

We will make the following notational conventions. If $\gamma:\left[0, t_{0}\right] \rightarrow \mathbb{C}$ or $\gamma:[0, \infty) \rightarrow \mathbb{C}$ is a curve and $t \leq t_{0}$, 
- We write $\gamma_{t}$ for the path $\gamma[0, t]$. The reference can either be to the function $\gamma:[0, t] \rightarrow \mathbb{C}$ or only to the trace $\gamma[0, t]$. For simple curves these are the same thing modulo increasing reparametrizations.

- We write $\gamma(t)$ for the point on the curve at time $t$.

- We write $\gamma$ for $\gamma\left[0, t_{0}\right]$ or $\gamma[0, \infty)$.

- If $D$ is a domain, we write $\gamma_{t} \subset D$ to mean $\gamma(0, t] \subset D$ and we write $\gamma \subset D$ if $\gamma\left(0, t_{0}\right) \subset D$.

If $z \in D, w \in \partial D$ and $\partial D$ is smooth near $w$, we let $h_{D}(z, w)$ be $\pi$ times the Poisson kernel. In other words, the probability that a Brownian motion starting at $z$ exits $D$ at a smooth arc $V \subset \partial D$ is given by

$$
\frac{1}{\pi} \int_{V} h_{D}(z, w)|d w|
$$

It is well known that

$$
h_{\mathbb{H}}(x+i y, 0)=\frac{y}{x^{2}+y^{2}}, \quad h_{\mathbb{D}}\left(0, e^{i \theta}\right)=\frac{1}{2} .
$$

Under conformal transformations, we have

$$
f \circ h_{D}(z, w)=\left|f^{\prime}(w)\right| h_{f(D)}(f(z), f(w)) .
$$

If $z, w$ are smooth boundary points, we write $h_{\partial D}(z, w)$ for $\pi$ times the boundary Poisson kernel, i.e., the inward normal derivative of $h_{D}(z, w)$ at $z$. It satisfies

$$
f \circ h_{\partial D}(z, w)=\left|f^{\prime}(z)\right|\left|f^{\prime}(w)\right| h_{\partial f(D)}(f(z), f(w)) .
$$

In other words, the Poisson kernel has boundary scaling exponent 1 and interior scaling exponent 0 . Two examples are

$$
h_{\partial \mathbb{H}}\left(x_{1}, x_{2}\right)=\left|x_{2}-x_{1}\right|^{-2}, \quad h_{\partial \mathbb{D}}(z, w)=|z-w|^{-2} .
$$

\subsection{Simply connected domains}

Suppose $D \subset \mathbb{H}$ is a simply connected subdomain with $\mathbb{H} \backslash D$ bounded and $\operatorname{dist}(0, \mathbb{H} \backslash D)>0$. Let $\Phi_{D}$ be the unique conformal transformation of $\mathbb{H} \backslash D$ onto $\mathbb{H}$ with $\Phi_{D}(z)=z+o(1)$ as $z \rightarrow \infty$. If $\gamma(t)$ is an $S L E_{\kappa}$ curve from 0 to infinity, let $\tau=\inf \{t: \operatorname{dist}(\gamma(t), \mathbb{H} \backslash D)=0\}$. If $t<\tau$, we let $D_{t}=g_{t}(D)$ and $\Phi_{t}=\Phi_{D_{t}}$. The fundamental local martingale introduced in [7] is

$$
M_{t}=\exp \left\{-\frac{a \mathbf{c}}{12} \int_{0}^{t} S \Phi_{s}\left(U_{s}\right) d s\right\} \Phi_{t}^{\prime}\left(U_{t}\right)^{b},
$$

where $S$ denotes the Schwarzian derivative. It satisfies

$$
d M_{t}=b\left[\log \Phi_{t}^{\prime}\left(U_{t}\right)\right]^{\prime} M_{t} d U_{t}, \quad t<\tau .
$$


If we weight by the local martingale $M_{t}$, the driving function $U_{t}$ satisfies

$$
d U_{t}=b\left[\log \Phi_{t}^{\prime}\left(U_{t}\right)\right]^{\prime} d t+d W_{t},
$$

where $W_{t}$ is a Brownian motion in the new measure. Using Itô's formula, one can show that this is the same drift as one gets by taking the conformal image of $S L E_{\kappa}$ in $\mathbb{H}$ onto $D$. In other words, $S L E_{\kappa}$ in $D$, at least up to time $\tau$, can be obtained by taking $S L E_{\kappa}$ in $\mathbb{H}$ and weighting locally by $\Phi_{t}^{\prime}\left(U_{t}\right)^{b}$. (Weighting locally by $\Phi_{t}^{\prime}\left(U_{t}\right)^{b}$, or any other sufficiently differentiable function, is defined to be the same as weighting by the corresponding local martingale; in this case the local martingale is $M_{t}$.)

We will use the interpretation of the exponential term of (3) in terms of a conformally invariant quantity known as the (Brownian) loop measure [8]. We refer to Section 4 for a definition of the loop measure, but we state that it is a $\sigma$-finite measure on unrooted loops modulo reparametrization in $\mathbb{C}$. It satisfies the following properties.

- Restriction. If $D \subset \mathbb{C}$, the loop measure in $D$ is the loop measure in $\mathbb{C}$ restricted to loops in $D$.

- Conformal invariance If $f: D \rightarrow f(D)$ is a conformal transformation, then the image of the loop measure on $D$ under $f$ is the same as the loop measure in $f(D)$.

These properties characterize the loop measure as a measure on outer boundaries or filled "hulls" but not in general; for example, one can start with the outer boundaries of the loop measure in $\mathbb{C}$ and then define in other domains by restriction. This also gives a family satisfying these conditions [11]. The definition of the loop measure is up to a mutliplicative constant which is chosen so that the natural random walk loop measure approaches the Brownian loop measure.

We call a set $K \subset \mathbb{C}$ nonpolar if it is hit by Brownian motion with positive probability (and hence with probability one). We write $\Lambda\left(K_{1}, K_{2} ; D\right)$ for the measure of the set of loops in $D$ that intersect both $K_{1}$ and $K_{2}$. If $K_{1}$ is bounded, $\operatorname{dist}\left(K_{1}, K_{2}\right)>0$, and $\partial D$ is nonpolar, then $\Lambda\left(K_{1}, K_{2} ; D\right)<\infty$. Also, if $f: D \rightarrow f(D)$ is a conformal transformation, then

$$
\Lambda\left(f\left(K_{1}\right), f\left(K_{2}\right) ; f(D)\right)=\Lambda\left(K_{1}, K_{2} ; D\right) .
$$

If $K_{1}=\gamma_{t}$ is a curve in $D$ with $\gamma(0+)=0$, with corresponding conformal maps $g_{t}$ whose parametrization is chosen as in (2), then

$$
\Lambda\left(\gamma_{t}, \mathbb{H} \backslash D ; \mathbb{H}\right)=-\frac{a}{6} \int_{0}^{t} S \Phi_{s}\left(U_{s}\right) d s .
$$

The $a$ occurs because of the parametrization. If we had chosen the parametrization as in (2), the $a$ would be replaced with 2 .

If we define

$$
\Psi_{t, D}(\gamma)=\Psi\left(\gamma_{t}, \mathbb{H} \backslash D ; \mathbb{H}\right):=\exp \left\{\Lambda\left(\gamma_{t}, \mathbb{H} \backslash D ; \mathbb{H}\right)\right\}
$$


and let

$$
H_{D}(x, \infty)=\Phi_{D}^{\prime}(x)^{b}
$$

then the local martingale becomes

$$
M_{t}=\Psi_{t, D}(\gamma)^{\mathbf{c} / 2} H_{D_{t}}\left(U_{t}, \infty\right)
$$

We can think of $M_{t}$ as a function of the curve $\gamma_{t}$ rather than of the driving function $\left\{U_{s}\right.$ : $0 \leq s \leq t\}$. It can be shown in a straightforward manner that if $\gamma:[0, \infty) \rightarrow \mathbb{H}$ is a simple curve with $\gamma(0)=0, \gamma \subset D$ and $\gamma(t) \rightarrow \infty$ as $t \rightarrow \infty$, then $M_{t}(\gamma)$ is continuous, stays bounded, and

$$
\lim _{t \rightarrow \infty} M_{t}(\gamma)=\Psi_{\infty, D}(\gamma)^{\mathbf{c} / 2}
$$

Since $S L E_{\kappa}$ in $D$ satisfies these conditions for $\kappa \leq 4$, one can use the Girsanov Theorem (see [6]) to conclude that $T=\infty$ with probability one in the weighted measure and the following.

Proposition 2.1. For $\kappa \leq 4$, if

$$
M_{\infty}=\lim _{t \rightarrow \infty} M_{t}=1\{\gamma \subset D\} \Psi_{\infty, D}(\gamma)^{\mathbf{c} / 2}
$$

then

$$
\mathbf{E}\left[M_{\infty}\right]=M_{0}=\Phi_{D}^{\prime}(0)^{b} .
$$

This was proved in [7] for $\kappa \leq 8 / 3$ (for which $\mathbf{c} \leq 0$ ) using a more complicated argument. For $8 / 3<\kappa \leq 4$ (c $>0), M_{t}$ is not bounded and the Girsanov argument is the only way I know how to prove this. This argument does not extend to multiply connected domains, and some of the open problems in this paper deal with this issue.

\subsection{Chordal $S L E$ as a nonprobability measure}

It is useful to view $S L E$ as a measure that is not a probability measure. Let us define $\mu_{\mathbb{H}}(0, \infty)$ to be the probability measure $\mu_{\mathbb{H}}^{\#}(0, \infty)$ and if $D$ is a domain as in the previous section,

$$
\mu_{D}(0, \infty)=H_{D}(0, \infty) \mu_{D}^{\#}(0, \infty)
$$

where $H_{D}(0, \infty)$ is the "partition function" $\Phi_{D}^{\prime}(0)^{b}$. If $D_{1}, D_{2}$ are two such domains and $f: D_{1} \rightarrow D_{2}$ is a conformal transformation with $f(0)=0$ and $f(z) \sim z$ as $z \rightarrow \infty$, then

$$
f \circ \mu_{D_{1}}(0, \infty)=\left|f_{D_{1}}^{\prime}(0)\right|^{b} \mu_{D_{2}}(0, \infty) .
$$

If $f(z) \sim r z$ as $z \rightarrow \infty$, we will write $f^{\prime}(\infty)=1 / r$. This allows us to write the more general relation

$$
f \circ \mu_{D_{1}}(0, \infty)=\left|f_{D_{1}}^{\prime}(0)\right|^{b}\left|f_{D_{1}}^{\prime}(\infty)\right|^{b} \mu_{D_{2}}(0, \infty) .
$$

If $D$ is a simply connected domain, $\partial D$ is smooth near $w \in \partial D$ (we will call $w$ a smooth boundary point of $D$ ), and $f: D \rightarrow \mathbb{H}$ is a conformal transformation with $f(w)=\infty$, 
we write $f^{\prime}(w)=\left[f^{-1}\right]^{\prime}(\infty)=F^{\prime}(w)$ where $F(w)=1 / f(w)$. This allows us to define $\mu_{D}(z, w)=H_{D}(z, w) \mu_{D}^{\#}(z, w)$ for simply connected domains and smooth boundary points $z, w$ by conformal transformation using the conformal covariance rule

$$
f \circ \mu_{D}(z, w)=\left|f^{\prime}(z)\right|^{b}\left|f^{\prime}(w)\right|^{b} \mu_{f(D)}(f(z), f(w)) .
$$

A simple computation shows that $H_{\mathbb{H}}(x, y)=|x-y|^{-2 b}$ and more generally $H_{D}(x, y)=$ $h_{\partial D}(z, w)^{b}$ where $h_{\partial D}(z, w)$ is as in Section 2.1. If $\kappa \leq 4, D_{1} \subset D$, and $\partial D_{1}=\partial D$ near smooth boundary points $z, w$,

$$
\frac{d \mu_{D_{1}}(z, w)}{d \mu_{D}(z, w)}(\gamma)=1\left\{\gamma \subset D_{1}\right\} \Psi\left(\gamma_{t}, D \backslash D_{1} ; D\right)^{\mathbf{c} / 2}
$$

The quantity on the right is invariant under conformal transformations of $D$. For this reason, the quantity on the left can be defined even if the boundary is not smooth at $z, w$. Similarly, the ratio

$$
\frac{H_{D_{1}}(z, w)}{H_{D}(z, w)}
$$

is a conformal invariant and is well defined even for nonsmooth boundary points.

There is a way to view (4) in terms of partition functions. Since

$$
\frac{H_{D \backslash \gamma_{t}}(\gamma(t), \infty)}{H_{\mathbb{H} \backslash \gamma_{t}}(\gamma(t), \infty)}=\frac{H_{D_{t}}\left(U_{t}, \infty\right)}{H_{\mathbb{H}}\left(U_{t}, \infty\right)}=H_{D_{t}}\left(U_{t}, \infty\right)=\Phi_{t}^{\prime}\left(U_{t}\right)^{b},
$$

we see that

$$
\frac{d \mu_{D}(0, \infty)}{d \mu_{\mathbb{H}}(0, \infty)}\left(\gamma_{T}\right)=1\left\{\gamma_{T} \subset D\right\} \Psi_{T, D}(\gamma)^{\mathbf{c} / 2} \frac{H_{D \backslash \gamma_{T}}(\gamma(T), \infty)}{H_{\mathbb{H} \backslash \gamma_{T}}(\gamma(T), \infty)}
$$

This last equality holds under conformal transformation. If $D_{1} \subset D$ are simply connected domains, $z, w$ are smooth points of $\partial D$, and $D_{1}$ agrees with $D$ in neighborhoods of $z, w$, then

$$
\frac{d \mu_{D_{1}}(z, w)}{d \mu_{D}(z, w)}\left(\gamma_{T}\right)=1\left\{\gamma_{T} \subset D_{1}\right\} \Psi\left(\gamma_{T}, D \backslash D_{1} ; D\right)^{\mathbf{c} / 2} \frac{H_{D_{1} \backslash \gamma_{T}}(\gamma(T), w)}{H_{D \backslash \gamma_{T}}(\gamma(T), w)} .
$$

\section{$2.4 S L E_{\kappa}$ from 0 to $x$ in $\mathbb{H}$}

Let $x>0$. The measure $\mu_{\mathbb{H}}(0, x)$ can be studied by considering its Radon-Nikodym derivative with respect to $S L E_{\kappa}$ from 0 to $\infty$,

$$
M_{T}=\frac{d \mu_{\mathbb{H}}(0, x)}{d \mu_{\mathbb{H}}(0, \infty)}\left(\gamma_{T}\right) .
$$

Here $T$ is a stopping time. If $T=\infty$, these measures will be singular with respect to each other, so we will restrict to bounded stopping times $T$ with $\operatorname{dist}\left\{\gamma_{T},[x, \infty)\right\}>0$. Using the domain Markov property, we can write

$$
M_{T}=\frac{H_{\mathbb{H} \backslash \gamma_{T}}(\gamma(T), x)}{H_{\mathbb{H} \backslash \gamma_{T}}(\gamma(T), \infty)}
$$


Using the scaling relation we can write formally

$$
\frac{H_{\mathbb{H} \backslash \gamma_{T}}(\gamma(T), x)}{H_{\mathbb{H} \backslash \gamma_{T}}(\gamma(T), \infty)}=\frac{\left|g_{T}^{\prime}(\gamma(T))\right|^{b} g_{T}^{\prime}(x)^{b} H_{\mathbb{H}}\left(U_{T}, g_{T}(x)\right)}{\left|g_{T}^{\prime}(\gamma(T))\right|^{b} g_{T}^{\prime}(\infty)^{b} H_{\mathbb{H}}(0, \infty)} .
$$

If we cancel the identical terms $\left|g_{T}^{\prime}(\gamma(T))\right|^{b}$, not worrying about the fact that the derivative is not defined, we get the form of the local martingale

$$
M_{t}=g_{t}^{\prime}(x)^{b} H_{\mathbb{H}}\left(U_{t}, g_{t}(x)\right)=g_{t}^{\prime}(x)^{b}\left[g_{t}(x)-U_{t}\right]^{-2 b}
$$

If one is uncomfortable with the argument, one can just check directly that $M_{t}$ as defined in (9) is a local martingale satisfying

$$
d M_{t}=\frac{2 b}{g_{t}(x)-U_{t}} M_{t} d U_{t}
$$

If we weight by the local martingale, $U_{t}$ satisfies

$$
d U_{t}=\frac{2 b}{g_{t}(x)-U_{t}} d t+d W_{t}
$$

where $W_{t}$ is a standard Brownian motion in the new measure. In the usual parametrization of $S L E$, we could write this as

$$
d U_{\kappa t}=\frac{2 b \kappa}{g_{\kappa t}(x)-U_{\kappa t}} d t+d W_{\kappa t}
$$

This is an example of a $S L E(\kappa, \rho)$ process with

$$
\rho=2 b \kappa=6-\kappa .
$$

If $D$ is a domain as above, then we can write

$$
\frac{d \mu_{D}(0, x)}{d \mu_{\mathbb{H}}(0, \infty)}=\frac{d \mu_{D}(0, x)}{d \mu_{D}(0, \infty)} \frac{d \mu_{D}(0, \infty)}{d \mu_{\mathbb{H}}(0, \infty)}
$$

If $\gamma_{T} \subset D$ and we write $x_{t}=g_{t}(x)$,

$$
\begin{aligned}
\frac{d \mu_{D}(0, x)}{d \mu_{D}(0, \infty)}\left(\gamma_{T}\right) & =\frac{H_{D \backslash \gamma_{T}}(\gamma(T), x)}{H_{D \backslash \gamma_{T}}(\gamma(T), \infty)} \\
& =\frac{\left|g_{T}^{\prime}(\gamma(T))\right|^{b} g_{T}^{\prime}(x)^{b} H_{g_{T}(D)}\left(U_{T}, x_{T}\right)}{\left|g_{T}^{\prime}(\gamma(T))\right|^{b}\left|g_{T}^{\prime}(\infty)\right|^{b} H_{g_{T}(D)}\left(U_{T}, \infty\right)} \\
& =\frac{g_{T}^{\prime}(x)^{b} \Phi_{T}^{\prime}\left(U_{T}\right)^{b} \Phi_{T}^{\prime}\left(x_{T}\right)^{b} H_{\mathbb{H}}\left(\Phi_{T}\left(U_{T}\right), \Phi_{T}\left(x_{T}\right)\right)}{\Phi_{T}^{\prime}\left(U_{T}\right)^{b} \Phi_{T}^{\prime}(\infty)^{b} H_{\mathbb{H}}(0, \infty)} \\
& =g_{T}^{\prime}(x)^{b} \Phi_{T}^{\prime}\left(x_{T}\right)^{b}\left[\Phi_{T}\left(x_{T}\right)-\Phi_{T}\left(U_{T}\right)\right]^{-2 b} \\
& =F_{T}^{\prime}(x)^{b}\left[F_{T}(x)-F_{T}(\gamma(T))\right]^{-2 b},
\end{aligned}
$$


where $F_{T}=\Phi_{T} \circ g_{T}$ which is the conformal transformation of $D \backslash \gamma_{T}$ onto $\mathbb{H}$ with $F(z)=$ $z+o(1)$ as $z \rightarrow \infty$. From the previous section, if $\gamma_{T} \subset D$, we have

$$
\frac{d \mu_{D}(0, \infty)}{d \mu_{\mathbb{H}}(0, \infty)}\left(\gamma_{T}\right)=\Psi_{T, D}(\gamma)^{\mathbf{c} / 2} \frac{H_{D \backslash \gamma_{T}}(\gamma(T), \infty)}{H_{\mathbb{H} \backslash \gamma_{T}}(\gamma(T), \infty)}=\Psi_{T, D}(\gamma)^{\mathbf{c} / 2} \Phi_{T}^{\prime}\left(U_{T}\right)^{b}
$$

Therefore,

$$
\begin{aligned}
\frac{d \mu_{D}(0, x)}{d \mu_{\mathbb{H}}(0, \infty)}\left(\gamma_{T}\right) & =1\left\{\gamma_{T} \subset D\right\} \Psi_{T, D}(\gamma)^{\mathbf{c} / 2} \frac{H_{D \backslash \gamma_{T}}(\gamma(T), x)}{H_{\mathbb{H} \backslash \gamma_{T}}(\gamma(T), \infty)} \\
& =1\left\{\gamma_{T} \subset D\right\} \Psi_{T, D}(\gamma)^{\mathbf{c} / 2} F_{T}^{\prime}(x)^{b}\left[F_{T}(x)-F_{T}(\gamma(T))\right]^{-2 b} \Phi_{T}^{\prime}\left(U_{T}\right)^{b}
\end{aligned}
$$

\subsection{Radial $S L E_{\kappa}$}

If $D$ is a simply connected domain, $z$ a smooth boundary point of $\partial D$ and $w \in D$, then the radial $S L E_{\kappa}$ partition function is defined by $H_{\mathbb{H}}(0, i)=1$ and otherwise by the scaling rule

$$
H_{D}(z, w)=\left|f^{\prime}(z)\right|^{b}\left|f^{\prime}(w)\right|^{\tilde{b}} H_{f(D)}(f(z), f(w)) .
$$

In particular, if $f: D \rightarrow \mathbb{H}$ is the conformal transformation with $f(z)=i, f(w)=0$,

$$
H_{D}(z, w)=\left|f^{\prime}(z)\right|^{b}\left|f^{\prime}(w)\right|^{\tilde{b}}=\left|f^{\prime}(w)\right|^{\tilde{b}} h_{D}(w, z)^{b}
$$

where $h_{D}(w, z)$ is as in Section 2.1. (The partition function is determined up to an arbitrary multiplicative constant. We have chosen the constant so that $H_{\mathbb{H}}(0, i)=1$. Another possibility would be to choose $H_{\mathbb{D}}(1,0)=1$.)

The corresponding probability measure $\mu_{D}^{\#}(z, w)$ satisfies the domain Markov property (from the boundary): given an initial segment $\gamma_{t}$ starting at $z$, the conditional distribution of the remainder of the path is that of $\mu_{D \backslash \gamma_{t}}(\gamma(t), w)$.

If $D$ is a simply connected subdomain of $\mathbb{H}$ as in Section 2.2 and $w \in \mathbb{H}$, then we can consider the Radon-Nikodym derivative

$$
\frac{d \mu_{D}(0, w)}{d \mu_{\mathbb{H}}(0, \infty)}=\frac{d \mu_{D}(0, w)}{d \mu_{D}(0, \infty)} \frac{d \mu_{D}(0, \infty)}{d \mu_{\mathbb{H}}(0, \infty)}
$$

If $\gamma_{T} \subset D$, and we write $w_{t}=g_{t}(w), F_{t}=\Phi_{t} \circ g_{t}$, we get

$$
\begin{aligned}
\frac{d \mu_{D}(0, w)}{d \mu_{D}(0, \infty)}\left(\gamma_{T}\right) & =\frac{H_{D \backslash \gamma_{T}}(0, w)}{H_{D \backslash \gamma_{T}}(0, \infty)} \\
& =\frac{\left|g_{T}^{\prime}(\gamma(T))\right|^{b}\left|g_{T}^{\prime}(w)\right|^{\tilde{b}} H_{g_{T}(D)}\left(U_{T}, w_{T}\right)}{\left|g_{T}^{\prime}(\gamma(T))\right|^{b}\left|g_{T}^{\prime}(\infty)\right|^{b} H_{g_{T}(D)}\left(U_{T}, \infty\right)} \\
& =\left|F_{T}^{\prime}(w)\right|^{\tilde{b}} H_{\mathbb{H}}\left(F_{T}(\gamma(T)), F_{T}(w)\right) .
\end{aligned}
$$


Therefore,

$$
\begin{aligned}
\frac{d \mu_{D}(0, w)}{d \mu_{\mathbb{H}}(0, \infty)}\left(\gamma_{T}\right) & =1\left\{\gamma_{T} \subset D\right\} \Psi_{T, D}(\gamma)^{\mathbf{c} / 2} \frac{H_{D \backslash \gamma_{T}}(\gamma(T), w)}{H_{\mathbb{H} \backslash \gamma_{T}}(\gamma(T), \infty)} \\
& =1\left\{\gamma_{T} \subset D\right\} \Psi_{T, D}(\gamma)^{\mathbf{c} / 2} \Phi_{T}^{\prime}\left(U_{T}\right)^{b}\left|F_{T}^{\prime}(w)\right|^{\tilde{b}} H_{\mathbb{H}}\left(F_{T}(\gamma(T)), F_{T}(w)\right) .
\end{aligned}
$$

It is standard to study radial $S L E_{\kappa}$ as a measure on curves from a boundary point $z$ of the unit disk $\mathbb{D}$ to the origin. Given $\gamma_{T}$, we let $g_{T}$ be the unique conformal transformation of $\mathbb{D} \backslash \gamma_{T}$ to $\mathbb{D}$ with $g_{T}(0)=0, g_{T}^{\prime}(0)>0$. In this case we can compare $\mu_{\mathbb{D}}(z, 0)$ with the chordal measure $\mu_{\mathbb{D}}(z, w)$ where $w$ is another point in $\partial \mathbb{D}$. Note that $H_{\mathbb{D}}(z, w)=h_{\mathbb{D}}(z, w)^{b}=$ $|z-w|^{-2 b}$. Then if $w_{T}=g_{T}(w), U_{T}=g_{T}(\gamma(T))$,

$$
\begin{aligned}
\frac{d \mu_{\mathbb{D}}(z, 0)}{d \mu_{\mathbb{D}}(z, w)}\left(\gamma_{T}\right) & =\frac{H_{\mathbb{D} \backslash \gamma_{T}}(\gamma(T), 0)}{H_{\mathbb{D} \backslash \gamma_{T}}(\gamma(T), w)} \\
& =\frac{\left|g_{T}^{\prime}(\gamma(T))\right|^{b}\left|g_{T}^{\prime}(0)\right|^{\tilde{b}} H_{\mathbb{D}}\left(U_{T}, 0\right)}{\left|g_{T}^{\prime}(\gamma(T))\right|^{b}\left|g_{T}^{\prime}(w)\right|^{b} H_{\mathbb{D}}\left(U_{T}, w_{T}\right)} \\
& =g_{T}^{\prime}(0)^{\tilde{b}}\left|g_{T}^{\prime}(w)\right|^{-b}\left|w_{T}-U_{T}\right|^{2 b} H_{\mathbb{D}}(1,0) .
\end{aligned}
$$

Of course we can determine $H_{\mathbb{D}}(1,0)$, but we have written it this way to emphasize that the constant chosen in the definition of the radial partition function carries over to this formula. Here we have not discussed the parametrization. It is standard to parametrize radial $S L E_{\kappa}$ so that $g_{t}^{\prime}(0)=e^{t}$ in which case we write $g_{T}^{\prime}(0)^{\tilde{b}}=e^{\tilde{b} T}$.

Suppose $D$ is a simply connected subdomain of $\mathbb{D}$ including 0 and such that $D$ agrees with $\mathbb{D}$ in a neighborhood of $z \in \partial \mathbb{D}$. Let $\Phi_{T}$ be the conformal transformation that maps $g_{T}(D)$ onto $\mathbb{D}$ with $\Phi_{T}(0)=0, \Phi_{T}^{\prime}(0)>0$. Let $F_{T}=\Phi_{T} \circ g_{T}$ which is the conformal transformation that maps $D \backslash \gamma_{T}$ onto $\mathbb{D}$ with $F_{T}(0)=0, F_{T}^{\prime}(0)>0$. Then, if we write $\Psi_{T}=\Psi\left(\gamma_{T}, \mathbb{D} \backslash D ; \mathbb{D}\right)$, we get

$$
\begin{aligned}
\frac{d \mu_{D}(z, 0)}{d \mu_{\mathbb{D}}(z, 0)}\left(\gamma_{T}\right) & =1\left\{\gamma_{T} \subset D\right\} \Psi_{T}^{\mathbf{c} / 2} \frac{H_{D \backslash \gamma_{T}}(\gamma(T), 0)}{H_{D \backslash \gamma_{T}}(\gamma(T), 0)} \\
& =1\left\{\gamma_{T} \subset D\right\} \Psi_{T}^{\mathbf{c} / 2} \frac{H_{D_{T}}\left(U_{T}, 0\right)}{H_{\mathbb{D}}\left(U_{T}, 0\right)} \\
& =1\left\{\gamma_{T} \subset D\right\} \Psi_{T}^{\mathbf{c} / 2} \Phi_{T}^{\prime}(0)^{\tilde{b}}\left|\Phi_{T}^{\prime}\left(U_{T}\right)\right|^{b}
\end{aligned}
$$

As in the chordal case, we can let $T \rightarrow \infty$ and show that $\mu_{D}(z, 0)$ as a measure on the entire path $\gamma$ is absolutely continuous with respect to $\mu_{\mathbb{D}}(z, 0)$ with

$$
\frac{d \mu_{D}(z, 0)}{d \mu_{\mathbb{D}}(z, 0)}(\gamma)=1\left\{\gamma_{T} \subset D\right\} \Psi(\gamma ; \mathbb{D} \backslash D ; \mathbb{D})^{\mathbf{c} / 2}
$$

The partition function $H_{D}(z, 0)$ is the integral of the right-hand side with respect to the measure $\mu_{\mathbb{D}}(z, 0)$. 


\subsection{A slightly different measure}

Define the measure $\nu_{D, x}=\nu_{D, x ; \mathbb{H}}$ by

$$
\nu_{\mathbb{H}, x}\left[\gamma_{T}\right]=\mu_{\mathbb{H}}(0, \infty),
$$

and if $D$ is a subset as above and $T$ is a stopping time

$$
\frac{d \nu_{D, 0}}{d \nu_{\mathbb{H}, 0}}\left(\gamma_{T}\right)=\Psi_{T, D}^{\mathbf{c} / 2} 1\left\{\gamma_{T} \subset D\right\} .
$$

Roughly speaking, the measure $\nu_{\mathbb{H}, 0}$ is a measure on curves starting at 0 that are known to continue after time $T$, but the exact distribution of the remainder of the path is not specified. More generally, $\nu_{D_{1}, z ; D}$ is defined using the conformal covariance relation

$$
f \circ \nu_{D_{1}, z ; D}=\left|f^{\prime}(z)\right|^{b} \nu_{f\left(D_{1}\right), f(w) ; f(D)} .
$$

Here $f$ is a conformal transformation of the larger domain $D$. This defines the measure for smooth boundary points $z$. We do not define it for nonsmooth points although we can take ratios

$$
\frac{d \nu_{D_{1}, z ; D}}{d \nu_{D, z ; D}}
$$

if $D, D_{1}$ agree in neighborhoods of $z$.

\subsection{Multiple curves}

We can extend the definition of the measure of the last section to $n$-tuples of curves $\bar{\gamma}=$ $\left(\gamma^{1}, \ldots, \gamma^{n}\right)$ in $\mathbb{H}$ started at distinct real numbers $\mathbf{x}=\left(x_{1}, x_{2}, \ldots, x_{n}\right)$. If $\bar{t}=\left(t_{1}, \ldots, t_{n}\right)$ we write $\bar{\gamma}_{\bar{t}}$ for $\left(\gamma_{t_{1}}^{1}, \ldots, \gamma_{t_{n}}^{n}\right)$. Let $\bar{T}=\left(T_{1}, \ldots, T_{n}\right)$ be a stopping time for the $n$-tuple of processes. By this we mean that for each $t_{1}, \ldots, t_{n}$, the event $\left\{T_{1} \leq t_{1}, \ldots, T_{n} \leq t_{n}\right\}$ depends only on $\bar{\gamma}_{\bar{t}}$. We define $\nu_{\mathbb{H}, \mathbf{x}}$ by saying its Radon-Nikodym derivative with respect to the product measure

$$
\tilde{\mu}_{\mathbb{H}}(\mathbf{x}, \infty):=\mu_{\mathbb{H}}\left(x_{1}, \infty\right) \times \cdots \times \mu_{\mathbb{H}}\left(x_{n}, \infty\right) .
$$

is given by

$$
Y_{\mathbf{c}}\left(\bar{\gamma}_{\bar{T}}\right)=\frac{d \nu_{\mathbb{H}, \mathbf{x}}}{d \tilde{\mu}_{\mathbb{H}, \mathbf{x}}}\left(\bar{\gamma}_{\bar{T}}\right)=\prod_{j=2}^{n} \frac{d \nu_{\mathbb{H} \backslash\left(\gamma^{1} \cup \cdots \cup \gamma^{j-1}\right), x_{j}}}{d \mu_{\mathbb{H}}\left(x_{j}, \infty\right)}\left(\gamma_{T^{j}}^{j}\right) .
$$

This may appear to depend on the order in which the curves are written, but there is another way of writing the derivative that shows symmetry:

$$
Y_{\mathbf{c}}\left(\bar{\gamma}_{\bar{T}}\right)=1\left\{\gamma_{T_{j}}^{j} \cap \gamma_{T_{k}}^{k}=\emptyset, 1 \leq j<k \leq n\right\} \Psi\left(\bar{\gamma}_{\bar{T}}\right)^{\mathbf{c} / 2},
$$

where

$$
\Psi\left(\bar{\gamma}_{\bar{T}}\right)=\exp \left\{\sum_{j=2}^{n} m_{j}\left(\bar{\gamma}_{\bar{T}}, \mathbb{H}\right)\right\},
$$


and $m_{j}\left(\bar{\gamma}_{\bar{T}}, \mathbb{H}\right)$ denotes the loop measure of the set of loops that intersect at least $j$ of the $n$ paths $\gamma_{T_{1}}^{1}, \ldots, \gamma_{T^{n}}^{n}$. If $T_{j}=0$ for $j \geq 2$, then $Y_{\mathbf{c}}\left(\bar{\gamma}_{\bar{T}}\right)=1$.

Suppose we have a partition function $\hat{H}_{D}(\mathbf{z} ; \mathbf{w})$. Here $\mathbf{z}=\left(z_{1}, \ldots, z_{n}\right)$ are distinct smooth boundary points of $D$ and $\mathbf{w}=\left(w_{1}, \ldots, w_{l}\right)$ are other points all of which are distinct and are either smooth boundary points or interior points. We assume that there exist real numbers $\bar{\beta}=\left(\beta_{1}, \ldots, \beta_{l}\right)$ such that

$$
\hat{H}_{D}(\mathbf{z} ; \mathbf{w})=\left[\prod_{j=1}^{n}\left|f^{\prime}\left(z_{j}\right)\right|^{b}\right]\left[\prod_{k=1}^{l}\left|f^{\prime}\left(w_{k}\right)\right|^{\beta_{k}}\right] \hat{H}_{f(D)}(f(\mathbf{z}), f(\mathbf{w})) .
$$

(There may also be some implicit dependencies, e.g., giving some arcs "reflecting" conditions but we assume that these are conformally invariant.) One possibility is that $l=n$ and $\mathbf{w}=\left(w_{1}, \ldots, w_{n}\right)$ are "target" points for the paths. In this case, we let $\beta_{j}=b$ or $\tilde{b}$ respectively, if $w_{j}$ is a boundary point or an interior point. The Radon-Nikodym derivative of the corresponding measure $\hat{\mu}_{\mathbb{H}}(\mathbf{x}, \mathbf{w})$ with respect to $\tilde{\mu}_{\mathbb{H}}(\mathbf{x}, \infty)$ can be written as

$$
Y\left(\bar{\gamma}_{\bar{T}}\right) \frac{\hat{H}_{\mathbb{H} \backslash \bar{\gamma}_{\bar{T}}}(\mathbf{z} ; \mathbf{w})}{H_{\mathbb{H} \backslash \gamma_{T_{1}}^{1}}\left(z_{1}, \infty\right) \cdots H_{\mathbb{H} \backslash \gamma_{T_{n}}^{n}}\left(z_{n}, \infty\right)}, \quad z_{j}=\gamma^{j}\left(T_{j}\right)
$$

Let $g_{t}^{j}$ denote the conformal transformation of $\mathbb{H} \backslash \gamma_{t}^{j}$ onto $\mathbb{H}$ with $g_{t}^{j}(z)=z+o(1)$ as $z \rightarrow \infty$ and let $\bar{g}_{\bar{t}}$ denote the corresponding transformation for $\bar{\gamma}_{\bar{t}}$. Let

$$
\bar{V}_{\bar{t}}=\left(V_{\bar{t}}^{1}, \ldots, V_{\bar{t}}^{n}\right)=\left(\bar{g}_{\bar{T}}\left(\gamma^{1}\left(t_{1}\right)\right), \ldots, \bar{g}_{\bar{T}}\left(\gamma^{n}\left(t_{n}\right)\right)\right)
$$

The scaling relation implies that we can write (at least formally)

$$
\frac{\hat{H}_{\mathbb{H} \backslash \bar{\gamma}_{\bar{T}}}(\mathbf{z} ; \mathbf{w})}{H_{\mathbb{H} \backslash \gamma_{T_{1}}^{1}}\left(z_{1}, \infty\right) \cdots H_{\mathbb{H} \backslash \gamma_{T_{n}}^{n}}\left(z_{n}, \infty\right)}=H_{\mathbb{H}}\left(\bar{V}_{\bar{T}} ; \bar{g}_{\bar{T}}(\mathbf{w})\right)\left[\prod_{j=1}^{n} \frac{\left|\bar{g}_{\bar{T}}^{\prime}\left(z_{j}\right)\right|^{b}}{\left|\left(g_{T_{j}}^{j}\right)^{\prime}\left(z_{j}\right)\right|^{b}}\right]\left[\prod_{k=1}^{l}\left|\bar{g}_{\bar{T}}^{\prime}\left(w_{k}\right)\right|^{\beta_{k}}\right] .
$$

If we define $F_{\bar{t}}^{j}$ by $\bar{g}_{\bar{t}}=F_{\bar{t}}^{j} \circ g_{t_{j}}^{j}$ and let $U_{t}^{j}=g_{t}^{j}\left(z_{j}\right)$, we get the expression

$$
\frac{d \hat{\mu}_{\mathbb{H}}(\mathbf{x} ; \mathbf{w})}{d \tilde{\mu}_{\mathbb{H}}(\mathbf{x}, \infty)}\left(\bar{\gamma}_{T}\right)=Y\left(\bar{\gamma}_{\bar{T}}\right) H_{\mathbb{H}}\left(\bar{V}_{\bar{T}} ; \bar{g}_{\bar{T}}(\mathbf{w})\right)\left[\prod_{j=1}^{n}\left|\left(F_{\bar{T}}^{j}\right)^{\prime}\left(U_{T_{j}}^{j}\right)\right|^{b}\right]\left[\prod_{k=1}^{l}\left|\bar{g}_{\bar{T}}^{\prime}\left(w_{k}\right)\right|^{\beta_{k}}\right] .
$$

This formula becomes nicer if we allow only one of the curves to move. If only the first path moves, then: $T^{j}=0$ for $j \geq 2 ; \bar{g}_{\bar{T}}=g_{T^{1}}^{1} ; F_{\bar{T}}^{1}$ is the identity; and $F_{\bar{T}}^{j}=g_{T^{1}}^{1}$ for $j \geq 2$. Then,

$$
\frac{d \hat{\mu}_{\mathbb{H}}(\mathbf{x}, \mathbf{w})}{d \mu_{\mathbb{H}}\left(x_{1}, \infty\right)}\left(\gamma_{T_{1}}^{1}\right)=H_{\mathbb{H}}\left(\bar{V}_{\bar{T}} ; g_{T_{1}}^{1}(\mathbf{w})\right)\left[\prod_{j=2}^{n}\left|\left(g_{T_{1}}^{1}\right)^{\prime}\left(x_{j}\right)\right|^{b}\right]\left[\prod_{k=1}^{l}\left|\left(g_{T_{1}}^{1}\right)^{\prime}\left(w_{k}\right)\right|^{\beta_{k}}\right] \text {. }
$$




\subsubsection{Two paths going toward each other}

The first step in Zhan's proof of the reversibility of $S L E_{\kappa}[12]$ considers pairs of paths starting at $x_{1}, x_{2}$ heading toward each other. As mentioned in Section 2.4, $H_{\mathbb{H}}\left(x_{1}, x_{2}\right)=\left|x_{2}-x_{1}\right|^{-2 b}$. This is an example with $n=2$ and $l=0$. The Radon-Nikodym derivative (11) becomes

$$
\frac{d \hat{\mu}_{\mathbb{H}}(\mathbf{x})}{d \tilde{\mu}_{\mathbb{H}}(\mathbf{x}, \infty)}\left(\bar{\gamma}_{T}\right)=Y\left(\bar{\gamma}_{\bar{T}}\right)\left|V_{\bar{T}}^{2}-V_{\bar{T}}^{1}\right|^{-2 b}\left|\left(F_{\bar{T}}^{1}\right)^{\prime}\left(U_{T_{1}}^{1}\right)\right|^{b}\left|\left(F_{\bar{T}}^{2}\right)^{\prime}\left(U_{T_{2}}^{2}\right)\right| .
$$

There is much more to the proof of reversibility but we do not discuss it here.

\subsubsection{Non-intersecting paths}

We describe a slight generalization of the configurational measure discussed in [3]. Suppose $\mathbf{z}=\left(z_{1}, \ldots, z_{n}\right)$ are distinct smooth boundary points of simply connected $D$ and $\mathbf{w}=\left(w_{1}, \ldots, w_{n}\right)$ are $n$ other points in $\bar{D}$. The points $w_{1}, \ldots, w_{n}$ can be boundary or interior points, but we impose the topological condition that there exist nonintersecting paths $\gamma^{1}, \ldots, \gamma^{n}$ in $D$ with $\gamma^{j}$ connecting $z_{j}$ to $w_{j}$. We define the partition function $H_{D}(\mathbf{z}, \mathbf{w})$ and measure $\mu_{D}(\mathbf{z}, \mathbf{w})$ by induction on $n$. It will satisfy the conformal covariance condition

$$
H_{D}(\mathbf{z}, \mathbf{w})=\left[\prod_{j=1}^{n}\left|f^{\prime}\left(z_{j}\right)\right|^{b}\right]\left[\prod_{j=1}^{n}\left|f^{\prime}\left(w_{j}\right)\right|^{b_{j}}\right] H_{f(D)}(f(\mathbf{z}), f(\mathbf{w})),
$$

where $b_{j}=b$ or $\tilde{b}$, respectively, depending if $w_{j}$ is an interior or a boundary point.

- If $n=1, H_{D}(z, w)$ is the usual chordal or radial $S L E_{\kappa}$ partition function, depending on whether $w$ is an interior or a boundary point. We have the measure

$$
\mu_{D}(z, w)=H_{D}(z, w) \mu_{D}^{\#}(z, w) .
$$

- Suppose the partition function and measure have been defined for $n-1$. For any path $\gamma^{n} \subset D$ connecting $z_{n}$ to $w_{n}$, let

$$
Z\left(\gamma^{n}\right)=H_{D \backslash \gamma^{n}}(\hat{\mathbf{z}}, \hat{\mathbf{w}}) .
$$

where $\hat{\mathbf{z}}=\left(z_{1}, \ldots, z_{n-1}\right)$ and $\hat{\mathbf{w}}=\left(w_{1}, \ldots, w_{n-1}\right)$. Then $H_{D}(\mathbf{z}, \mathbf{w})$ is the integral of $Z\left(\gamma^{n}\right)$ with respect to the measure $\mu_{D}\left(z_{n}, w_{n}\right)$.

- The marginal distribution on $\gamma^{n}$ of $\mu_{D}(\mathbf{z}, \mathbf{w})$ has Radon-Nikodym derivative $Z$ with respect to $\mu_{D}\left(z_{n}, w_{n}\right)$.

- Given $\gamma^{n}$, the conditional distribution of $\left(\gamma^{1}, \ldots, \gamma^{n-1}\right)$ is $\mu_{D \backslash \gamma^{n}}^{\#}(\hat{\mathbf{z}}, \hat{\mathbf{w}})$. 
In this case the measure $H_{D}(\mathbf{z}, \mathbf{w})$ is absolutely continuous with respect to the product measure

$$
\mu_{D}\left(z_{1}, w_{1}\right) \times \cdots \times \mu_{D}\left(z_{n}, w_{n}\right)
$$

with Radon-Nikodym derivative of the form (10). Using this form we can see that the measure is independent of the order that we write the pairing $\left(z_{1}, w_{1}\right), \ldots,\left(z_{n}, w_{n}\right)$. The partition function and measure do depend on the pairing.

One can also define a partition function $H(\mathbf{z} ; \mathbf{w})$ and a measure $\mu(\mathbf{z} ; \mathbf{w})$ where one does not specify the pairing. This is given by the sum of the partition function and measure over all possible pairings (with the measure and partition function equaling zero if a pairing does not satisfy the topological condition). We can also start with $2 n$ boundary points $z_{1}, \ldots, z_{2 n}$ and sum the partition function and measure over all complete pairings of the $2 n$ vertices. Here we grow $2 n$ paths.

Problem. The symmetry in the above construction was shown by the symmetry of the Radon-Nikodym derivative with respect to product measure. One can do the same construction for $\kappa>4$. For ease assume $n=2$ and $4<\kappa<8$. Let us choose a measure on paths $\left(\gamma^{1}, \gamma^{2}\right)$ connecting boundary points $\left(z_{1}, w_{1}\right),\left(z_{2}, w_{2}\right)$ in $D$.

- The marginal distribution on $\gamma^{1}$ with respect to $\mu_{D}\left(z_{1}, w_{1}\right)$ is given by $H_{D \backslash \gamma^{1}}\left(z_{2}, w_{2}\right)$. Here $D \backslash \gamma^{1}$ represents the connected component that contains $z_{2}$, w $w_{2}$ on the boundary. If $z_{2}, w_{2}$ are not boundary points of the same connected component of $D \backslash \gamma^{1}$, $H_{D \backslash \gamma^{1}}\left(z_{2}, w_{2}\right)=0$.

- Given $\gamma^{1}$, the distribution of $\gamma^{2}$ is given by $\mu_{D \backslash \gamma^{1}}^{\#}\left(z_{2}, w_{2}\right)$.

On the event $E=\left\{\gamma^{1} \cap \gamma^{2}=\emptyset\right\}$, this measure is absolutely continuous with respect to the product measure with a Radon-Nikodym derivative as above. In particular, on this event the measure does not depend on the order of $\gamma^{1}, \gamma^{2}$. However, the complement of $E$ has positive measure. The question is: does this measure depend on the order of $\gamma^{1}, \gamma^{2}$ ?

\subsubsection{Paths going to the same point}

The partition function for $n$ non-intersecting $S L E_{\kappa}$ paths going to the same point boundary point $w$ can be obtained from the previous measure by letting the points $w_{j}$ approach $w$. The partition function is

$$
H_{\mathbb{H}}(\mathbf{x} ; \infty)=\prod_{j<k}\left|x_{k}-x_{j}\right|^{a}, \quad a=2 / \kappa,
$$

combined with the scaling relation

$$
H_{D}(\mathbf{z}, w)=\left|f^{\prime}(\mathbf{z})\right|^{b}\left|f^{\prime}(w)\right|^{\xi(n)} H_{f(D)}(f(\mathbf{z}), f(w)) .
$$

were $f^{\prime}(\mathbf{z})$ is shorthand for $f^{\prime}\left(z_{1}\right) \cdots f^{\prime}\left(z_{n}\right)$ and $\xi(n)$ an intersection exponent that can be calculated. 


\section{$3 \quad$ Speculations and open problems}

\subsection{Multiply connected domains}

If $D$ is a simply connected domain of $\mathbb{H}$ with $\mathbb{H} \backslash D$ bounded and $\operatorname{dist}(0, \mathbb{H} \backslash D)>0$, the partition function is given by

$$
H_{D}(0, \infty)=\mathbf{E}\left[1\{\gamma \subset D\} \Psi(\gamma, \mathbb{H} \backslash D ; \mathbb{H})^{\mathbf{c} / 2}\right]
$$

The partition function is extended to other domains by the scaling rule

$$
H_{D}(z, w)=\left|f^{\prime}(z)\right|^{b}\left|f^{\prime}(w)\right|^{b} H_{f(D)}(f(z), f(w)) .
$$

One way to extend $H_{D}(z, w)$ to multiply connected domains where $z, w$ are smooth boundary points on the same component of $\partial D$ is to use (12). Assuming $H_{D}(0, \infty)<\infty$, we can define the measure

$$
\mu_{D}(0, \infty)=H_{D}(z, w) \mu_{D}^{\#}(0, \infty),
$$

by saying that $\mu_{D}^{\#}(0, \infty)$ is the usual $S L E_{\kappa}$ weighted by the partition function $H_{D}(z, w)$. Finiteness of $H_{D}(0, \infty)$ for $\kappa \leq 8 / 3$ is immediate since $\mathbf{c} \leq 0$; in fact, this shows that the quantity is monotone in $D$. For $\kappa=2, H_{D}(0, \infty)$ equals the probability that a Brownian excursion in $\mathbb{H}$ stays in $D$ and if $z, w$ are smooth boundary points, $H_{D}(z, w)=h_{D}(z, w)$; for other values of $\kappa$ we do not expect $H_{D}(z, w)=h_{D}(z, w)^{b}$ for multiply connected domains. For $\kappa=8 / 3, H_{D}(0, \infty)=\mathbf{P}\{\gamma \subset D\}$. The proof of finiteness for simply connected domains and $8 / 3<\kappa \leq 4$ uses the known form of the partition function $\Phi_{D}^{\prime}(0)^{b}$. We certainly expect this to be finite for this range of $\kappa$, but this is open.

Problem. Show that if $8 / 3<\kappa \leq 4$, and $D$ is a finitely connected subdomain of $\mathbb{H}$ with $\mathbb{H} \backslash D$ bounded and $\operatorname{dist}(0, \mathbb{H} \backslash D)>0$, then $H_{D}(0, \infty)$ as defined in (12) is finite. Is it monotone in $D$ ?

In order to define $\mu_{D}(z, w)$ where $z, w$ are in different components of $\partial D$, one needs to define the measure for at least one such case, say an annulus. Once this is defined, one can use an expression such as (12) to define it for all finitely connected $D$. The next section will discuss the case of an annulus.

\subsection{Annulus}

Let $\mathcal{A}_{r}$ denote the annulus $\mathcal{A}_{r}=\{r<|z|<1\}$ with boundary circles $C_{r}, C_{1}$ and let $z \in C_{1}, w \in C_{r}$. We will discuss the version of chordal $S L E_{\kappa}$ in $\mathcal{A}_{r}$ from $z$ to $w$ which is analogous to that discussed in the previous section. For ease we choose $z=1, w=r e^{i \theta}$. We restrict to $\kappa \leq 4$. 


\subsubsection{First approach}

If $\gamma:[0, t] \rightarrow \mathcal{A}_{r}$ is a simple curve with $\gamma(0)=z, \gamma(t)=w, \gamma(0, t) \subset \mathcal{A}_{r}$, then there exists a simply connected domain $D \subset \mathcal{A}_{r}$ that agrees with $\mathcal{A}_{r}$ in neighborhoods of $z, w$ and such that $\gamma \subset D$. Hence, in order to define the measure $\mu_{\mathcal{A}_{r}}(z, w)$ it suffices to define $\mu_{\mathcal{A}_{r}}(z, w ; D)$, the restriction of $\mu_{\mathcal{A}_{r}}(z, w)$ to curves with $\gamma \subset D$, for every simply connected domain $D \subset \mathcal{A}_{r}$ that agrees with $\mathcal{A}_{r}$ near $w, z$. We do so by stating that

$$
\frac{d \mu_{\mathcal{A}_{r}}(z, w ; D)}{d \mu_{D}(z, w)}(\gamma)=\Psi\left(\gamma, \mathcal{A}_{r} \backslash D ; \mathcal{A}_{r}\right)^{-\mathbf{c} / 2} .
$$

This is analogous to (5); the sign is changed because the numerator refers to the larger domain and the denominator to the subdomain. It is straightforward to show that this definition is consistent and hence gives a measure $\mu_{\mathcal{A}_{r}}(z, w)$. We define similarly $\mu_{A}(z, w)$ for any conformal annulus $A$ and smooth boundary points $z, w$ on different components of $\partial A$. This definition satisfies the conformal covariance rule

$$
f \circ \mu_{A}(z, w)=\left|f^{\prime}(z)\right|^{b}\left|f^{\prime}(w)\right|^{b} \mu_{f(A)}(f(z), f(w)) .
$$

This is easily verified by noting that for each subdomain $D$ as above

$$
f \circ \mu_{D}(z, w)=\left|f^{\prime}(w)\right|^{b}\left|f^{\prime}(z)\right|^{b} \mu_{D}(f(z), f(w))
$$

and using conformal invariance of the loop measure. It does not follow immediately that $\mu_{A}(z, w)$ is a finite measure, which we certainly expect to be true. For $8 / 3<\kappa \leq 4$, since c $>0$, it follow immediately that $\left|\mu_{A}(z, w ; D)\right|<\infty$ for each simply connected $D$. This is not immediate for $\kappa \leq 8 / 3$ by the above construction but the second approach below shows that for these $\kappa,\left|\mu_{A}(z, w)\right|<\infty$. Finiteness is needed to define the probability measure $\mu_{A}^{\#}(z, w)$. We define

$$
H_{A}(z, w)=\left|\mu_{A}(z, w)\right|,
$$

and note that $H_{A}$ satisfies the scaling rule

$$
H_{A}(z, w)=\left|f^{\prime}(z)\right|^{b}\left|f^{\prime}(w)\right|^{b} H_{f(A)}(f(z), f(w)) .
$$

Problem. Prove that $H_{\mathcal{A}_{r}}\left(1, r e^{i \theta}\right)<\infty$ if $8 / 3<\kappa \leq 4$.

In the special case $\kappa=2$, the partition function is known, $H_{A}(z, w)=h_{A}(z, w)$. This uses special properties of $\kappa=2$ related to the loop-erased random walk and should not be true for other values of $\kappa$.

\subsubsection{Second approach}

Let $w=r e^{i \theta}$ with $0 \leq \theta<2 \pi$. For each simple curve $\gamma$ connecting 1 to $w$ there is an integer $m=m(\gamma)$ such that the continuous argument $\theta(s)$ of $\gamma(s)$ with $\theta(0)=0$ equals $\theta+2 \pi m$ at the terminal point. We write

$$
\mu_{\mathcal{A}_{r}}(1, w)=\sum_{m=-\infty}^{\infty} \mu_{\mathcal{A}_{r}, m}(1, w)
$$


where $\mu_{\mathcal{A}_{r}, m}(1, w)$ denotes $\mu_{\mathcal{A}_{r}}\left(1, r e^{i \theta}\right)$ restricted to curves whose continuous argument is $\theta+2 \pi m$.

Let $V_{r}=\{x+i y: 0<y<\log r\}$ and $F(z)=e^{i z}$ which maps $V_{r}$ to $\mathcal{A}_{r}$. Note that $F$ is locally conformal but not globally one-to-one. If $\hat{z}=s e^{i \phi}$, the preimage of $\hat{z}$ under $F$ consists of all the points

$$
[\phi+2 \pi m]-i \log s, \quad m \in \mathbb{Z} .
$$

For each simple curve in $\mathcal{A}_{r} \gamma$ connecting 1 and $w$, let $\tilde{\gamma}$ be the continuous preimage of $\gamma$ under $F$; $\tilde{\gamma}$ is a simple curve in $V_{r}$ connecting 0 to $\tilde{w}=(\theta+2 \pi m)-i \log r$, where $m=m(\gamma)$. Similarly, if $D$ is a simply connected domain as above, there is a simply connected domain $\tilde{D}$ with $0 \in \partial \tilde{D}$ such that $F$ maps $\tilde{D}$ conformally onto $D$ with $F(0)=1, F(\theta+2 \pi m-i \log r)=w$. Let

$$
\nu_{m}(1, w ; D)=\nu_{\mathcal{A}_{r}, m}(1, w ; D)=r^{-b}\left[F \circ \mu_{\tilde{D}}(0,(\theta+2 \pi m)-i \log r)\right] .
$$

The factor $r^{-b}$ equals $\left|F^{\prime}(0)\right|^{-b}\left|F^{\prime}(\theta+2 \pi m-i \log r)\right|^{-b}$. Let $\nu_{m}(1, w)$ be the measure obtained by considering the consistent family $\nu_{m}(1, w ; D)$ over all $D$. Note that

$$
\nu_{m}(1, w)=\nu_{\mathcal{A}_{r}, m}(1, w)=r^{-b}\left[F \circ \mu_{V_{r}}^{*}(0, \theta+2 \pi m-i \log r)\right],
$$

where $\mu_{V_{r}}^{*}(0, x-i \log r)$ is $\mu_{V_{r}}(0, x-i \log r)$ restricted to curves $\tilde{\gamma}$ such that $F \circ \tilde{\gamma}$ is simple. Let $\nu_{\mathcal{A}_{r}}(1, w)=\sum_{m \in \mathbb{Z}} \nu_{m}(1, w)$. Since $\tilde{D}$ is a simply connected subdomain of the simply connected domain $V_{r}$, we know that

$$
\frac{d \mu_{\tilde{D}}(0,(\theta+2 \pi m)-i \log r)}{d \mu_{V_{r}}(0,(\theta+2 \pi m)-i \log r)}(\tilde{\gamma})=\Psi\left(\tilde{\gamma}, V_{r} \backslash \tilde{D} ; V_{r}\right)^{\mathbf{c} / 2}
$$

Similarly, we write

$$
\frac{d \mu_{D}(1, w)}{d \mu_{\mathcal{A}_{r}}(1, w)}(\gamma)=\Psi\left(\gamma, \mathcal{A}_{r} \backslash D ; \mathcal{A}_{r}\right)^{\mathbf{c} / 2} .
$$

Hence if $\gamma$ is a simple curve contained in simply connected $D$,

$$
\frac{d \mu_{\mathcal{A}_{r}}(1, w)}{d \nu_{\mathcal{A}_{r}}(1, w)}(\gamma)=\left[\frac{\Psi\left(\tilde{\gamma}, V_{r} \backslash \tilde{D} ; V_{r}\right)}{\Psi\left(\gamma, \mathcal{A}_{r} \backslash D ; \mathcal{A}_{r}\right)}\right]^{\mathbf{c} / 2} .
$$

To understand the right-hand side we need to consider the difference between the loop measure in $\mathcal{A}_{r}$ restricted to curves that intersect $\gamma$ and the image under $F$ of the loop measure in $V_{r}$ restricted to curves that intersect $\tilde{\gamma}$. In both cases we will explore the loop measure using the curve $\gamma$ or $\tilde{\gamma}$ starting at 1 or 0 , respectively. We will not explain this here in detail, but for every unrooted loop $\omega$ in $\mathcal{A}_{r}$ that intersects $\gamma$, there is a smallest $s$ such that $\gamma(s) \in \omega$. In the exploration process we "find" the unrooted loop $\omega$ at time $s$. It is associated to the corresponding rooted loop $\omega$ with root $\gamma(s)$ which is a "boundary bubble" in the domain $\mathcal{A}_{r} \backslash \gamma_{s}$. One similarly explores loops in $V_{r}$. There are two types of loops that appear in one of the measures but not the other. 
- If a loop in $\mathcal{A}_{r}$ has nonzero winding number about zero, it is not an image of a loop in $V_{r}$. Let $q(r)<\infty$ denote the total mass of the set of loops of nonzero winding number in $\mathcal{A}_{r}$. Topological considerations imply that every such loop intersects $\gamma$ and $\mathcal{A}_{r} \backslash D$. As $r \rightarrow 0, q(r) \sim[\log r] / 6$ (see Section 4$)$.

- If a loop rooted at $\tilde{\gamma}(s)$ in $V_{r}$ intersects one of the $2 \pi k$ translates of $\tilde{\gamma}_{s}$, then the image of this loop is not in the loop measure in $\mathcal{A}_{r}$. This is because at time $s$, the exploration process in $V_{r}$ is exploring loops in $V_{r} \backslash \tilde{\gamma}_{s}$ while the exploration process in $\mathcal{A}_{r}$ is exploring loops in $\mathcal{A}_{r} \backslash \gamma_{s}$. Loops in $V_{r} \backslash \tilde{\gamma}_{s}$ that intersect a translate of $\tilde{\gamma}_{s}$ do not correspond to loops in $\mathcal{A}_{r} \backslash \gamma_{s}$.

Suppose the argument $\theta(s)$ of $\gamma(s)$ is defined continuously. For each unrooted loop $\omega$ in $\mathcal{A}_{r}$, let $s$ be the smallest number with $\gamma(s) \in \omega$, and define the argument continuously on $\omega$ so that the argument agrees at $\gamma(s)$. Let $k=k(\omega, \gamma)$ be the number of nonzero integers $j$ such that there is an intersection of $\omega$ and $\gamma$ at which the argument of $\omega$ minus the argument of $\gamma$ equals $2 \pi k$. Let $\rho_{k}(\gamma)$ denotes the loop measure of the set of $\omega$ with $k(\omega, \gamma)=k$, and

$$
\beta(\gamma)=\sum_{k=1}^{\infty} k \rho_{k}(\gamma)
$$

$\beta(\gamma)$ is finite for each $\gamma$ but depends on $\gamma$. If $\gamma$ winds many times around the origin, then $\beta(\gamma)$ can be very large. We see that

$$
\frac{d \mu_{\mathcal{A}_{r}}(1, w)}{d \nu_{\mathcal{A}_{r}}(1, w)}(\gamma)=\exp \left\{\frac{\mathbf{c}}{2}[\beta(\gamma)-q(r)]\right\}
$$

Conformal transformation shows that $H_{V_{r}}(0, x+i r)$ decays exponentially in $x$. Using this, we get immediately that $H_{\mathcal{A}_{r}}(1, w)<\infty$ for $\kappa \leq 8 / 3$ since $\mathbf{c} \leq 0$. Although we strongly believe that it is true, it has not been proved that $H_{\mathcal{A}_{r}}\left(1, r e^{i \theta}\right)<\infty$ for $8 / 3<\kappa \leq 4$. Despite this fact, we will assume that it is true.

The rest of this section will be speculative assuming that $H_{\mathcal{A}_{r}}\left(1, r e^{i \theta}\right)$ is finite and $\mu_{\mathcal{A}_{r}}\left(1, r e^{i \theta}\right)^{\#}$ is well defined. If $|\theta|<\pi$, then as $r \rightarrow 1-$, we should have

$$
H_{\mathcal{A}_{r}}\left(1, r e^{i \theta}\right) \sim H_{\mathcal{A}_{r} \backslash(-r,-1)}\left(1, r e^{i \theta}\right) .
$$

The reason is that for $r$ near one, $H_{\mathcal{A}_{r}}\left(1, r e^{i \theta}\right)$ should be concentrated on paths that do not intersect the negative real axis. The asymptotics of $H_{\mathcal{A}_{r}}\left(1, r e^{i \theta}\right)$ as $r$ tends to zero are not as obvious. We certainly expect that the asymptotic value is independent of $\theta$. In Section 3.4 we give an argument to support the conjecture that

$$
H_{\mathcal{A}_{r}}(1, r) \sim c r^{\tilde{b}-b}[\log (1 / r)]^{\mathbf{c} / 2}, \quad r \rightarrow 0
$$

For $\kappa=2$ this can be verified by exact computation of $h_{\mathcal{A}_{r}}(1, r)$.

Problem. Prove or disprove (13) and (14). 


\subsection{Annulus and radial $S L E$}

Suppose $C$ is a simple closed curve surrounding the origin contained in the unit disk. Let $A$ denote the conformal annulus bounded by $C \cup \partial \mathbb{D}$ and suppose that $w$ is a smooth boundary point of $C$. Let $z \in \partial \mathbb{D}$. Let $\gamma$ denote a radial $S L E_{\kappa}$ curve from $z$ to 0 in $\mathbb{D}$ with conformal transformations $g_{t}$ parametrized so that $g_{t}^{\prime}(0)=e^{t}$. Let $\tau=\inf \{t: \gamma(t) \in C\}$. If $t<\tau$, we let $C_{t}=g_{t}(C), w_{t}=g_{t}(w)$, and $A_{t}$ the conformal annulus bounded by $\partial \mathbb{D}$ and $C_{t}$. Then if

$$
Y_{t}=1\{t<\tau\} \Psi\left(\gamma_{t}, C ; \mathbb{D}\right)^{\mathbf{c} / 2}
$$

we can write

$$
\begin{aligned}
\frac{d \mu_{A}(z, w)}{d \mu_{\mathbb{D}}(z, 0)}\left(\gamma_{T}\right) & =Y_{T} \frac{H_{A \backslash \gamma_{T}}(\gamma(T), w)}{H_{\mathbb{D} \backslash \gamma_{T}}(\gamma(T), 0)} \\
& =Y_{T} \frac{\left|g_{T}^{\prime}(\gamma(T))\right|^{b}\left|g_{T}^{\prime}(w)\right|^{b} H_{A_{T}}\left(U_{T}, w_{T}\right)}{\left|g_{T}^{\prime}(\gamma(T))\right|^{b}\left|g_{T}^{\prime}(0)\right|^{\tilde{b}} H_{\mathbb{D}}\left(U_{T}, 0\right)} \\
& =Y_{T} e^{-\tilde{b} T}\left|g_{T}^{\prime}(w)\right|^{b} H_{A_{T}}\left(U_{T}, w_{T}\right) H_{\mathbb{D}}(1,0)^{-1} .
\end{aligned}
$$

This should give a local martingale.

\subsection{Radial started from the interior}

We end with discussion of radial $S L E_{\kappa}$ from an interior point to a boundary point for $\kappa \leq 4$. To describe this we consider its Radon-Nikodym derivative with respect to whole plane $S L E_{\kappa}$. Whole plane $S L E$ as defined in [4] is a probability measure $\mu_{\mathbb{C}}=\mu_{\mathbb{C}}^{\#}(0, \infty)$ on paths $\gamma(-\infty, \infty)$ with $\gamma(-\infty)=0$. It is described in terms of the conformal maps

$$
F_{t}: \mathbb{C} \backslash \gamma[-\infty, t] \rightarrow \mathbb{C} \backslash \overline{\mathbb{D}}
$$

The parametrization and choice of the maps $F_{t}$ are such that

$$
F_{t}(z)=e^{-t} z+O(1), \quad z \rightarrow \infty
$$

It is defined so that it has the following property: given the path up to some time $t>-\infty$, the remainder of the path has the distribution of radial $S L E_{\kappa}$ from $\gamma(t)$ to $\infty$ in the domain $\mathbb{C} \backslash \gamma_{t}$.

If $T$ is a stopping time for whole plane $S L E$ such that $-\infty<T<\infty$, then $\mu_{\mathbb{C}}^{\#}$ can be considered as a measure $\mu_{\mathbb{C}, T}^{\#}$ on bounded curves $\gamma[-\infty, T]$. If $T=t$ is constant, and $f_{s}(z)=e^{s} z$, then we have the scaling rule

$$
f_{s} \circ \mu_{\mathbb{C}, t}^{\#}=\mu_{\mathbb{C}, t+s}^{\#}
$$

It is convenient to consider a measure that is not a probability measure. For any stopping time $T$ with $T>-\infty$, we define the measure, which we write as $\mu_{\mathbb{C}}$ (or $\mu_{\mathbb{C}, T}$ if we need to be 
explicit) whose Radon-Nikodym derivative with respect to $\mu_{\mathbb{C}, T}^{\#}$ is $e^{-\tilde{b} T}$. If $T=t$ is constant we get the conformal covariance rule

$$
f_{s} \circ \mu_{\mathbb{C}, t}=e^{\tilde{b} s} \mu_{\mathbb{C}, t+s}=\left|f_{s}^{\prime}(0)\right|^{\tilde{b}} \mu_{\mathbb{C}, t+s}
$$

When studying the Brownian loop measure in $\mathbb{C}$, one technical issue arise. If $K_{1}, K_{2}$ are two nonpolar sets, then $\Lambda\left(K_{1}, K_{2} ; \mathbb{C}\right)=\infty$. Indeed, if $K_{1}$ is bounded and nonpolar, one can show (see (24))

$$
\Lambda\left(K_{1}, \mathbb{D}_{2^{n+1}} \backslash \mathbb{D}_{2^{n}} ; \mathbb{D}_{2^{n}}\right)=\frac{1}{n}+O\left(n^{-2}\right), \quad n \rightarrow \infty .
$$

For large $n$, most loops in $\mathbb{D}_{2^{n+1}}$ that intersect both $K_{1}$ and $\mathbb{D}_{2^{n+1}} \backslash \mathbb{D}_{2^{n}}$ also intersect nonpolar $K_{2}$. In fact, the measure of the set of loops in $\mathbb{D}_{2^{n+1}}$ that intersect $\mathbb{D}_{2^{n+1}} \backslash \mathbb{D}_{2^{n}}$ and $K_{1}$ but do not intersect $K_{2}$ decays like $O\left(n^{-2}\right)$. This allows us to define $\Lambda^{*}\left(K_{1}, K_{2}\right)$ for bounded $K_{1}$ by

$$
\Lambda\left(K_{1}, K_{2} ; \mathbb{D}_{R}\right)=\log \log R+\Lambda^{*}\left(K_{1}, K_{2}\right)+o(1), \quad R \rightarrow \infty .
$$

$\Lambda^{*}$ is symmetric in $K_{1}, K_{2}$; invariant under rotations, dilations, and translations; and satisfies the following domain Markov property: if $K_{1} \subset K_{3}$, then

$$
\Lambda^{*}\left(K_{3}, K_{2}\right)=\Lambda^{*}\left(K_{1}, K_{2}\right)+\Lambda\left(K_{3} \backslash K_{1}, K_{2} ; \mathbb{C} \backslash K_{1}\right) .
$$

(There is some arbitrariness in the definition of $\Lambda^{*}$; for every $c_{1}, \Lambda+c_{1}$ satisfies the same properties.) If $K_{1}$ is polar, then $\Lambda\left(K_{1}, K_{2} ; \mathbb{D}_{R}\right)=0$ and hence $\Lambda^{*}\left(K_{1}, K_{2}\right)=-\infty$. If $K_{1} \cap K_{2}$ is nonpolar, then $\Lambda\left(K_{1}, K_{2} ; \mathbb{D}_{R}\right)=\infty$ for large $R$ and hence $\Lambda^{*}\left(K_{2}, K_{2}\right)=\infty$.

Using (15), one can show that as $r \rightarrow 0$,

$$
\Lambda^{*}(r \partial \mathbb{D}, \partial \mathbb{D})=-\log \log (1 / r)+O(1)
$$

Suppose $D$ is a domain containing 0 and $z$ is a smooth boundary point of $D$. Let $\tau=\inf \{t: \gamma(t) \in \partial D\}$. If $t<\tau$, let $D_{t}=F_{t}(D), z_{t}=F_{t}(z)$. Let $T$ be a stopping time with $T<\tau$. Then we define

$$
\frac{d \mu_{D}(0, z)}{d \mu_{\mathbb{C}}(0, \infty)}\left(\gamma_{T}\right)=c_{0} \exp \left\{\frac{\mathbf{c}}{2} \Lambda^{*}\left(\gamma_{T}, \partial D\right)\right\} 1\left\{\gamma_{T} \subset D\right\} H_{D_{T}}\left(U_{T}, z_{T}\right)\left|F_{T}^{\prime}(z)\right|^{b}
$$

The positive constant $c_{0}$ can be chosen arbitrarily although there should be a natural choice that is most convenient. The arbitrariness in the definition of $\Lambda^{*}$ can be handled by choosing $c_{0}$ appropriately so we will fix the definition of $\Lambda^{*}$ as above.

This definition has been chosen so that is satisfies the following domain Markov property,

- In the probability measure $\mu_{D}^{\#}(0, z)$ on paths $\gamma\left[0, t_{\gamma}\right]$, the conditional distribution of $\gamma\left[t, t_{\gamma}\right]$ given $\gamma_{t}$ is that of

$$
\mu_{D \backslash \gamma_{t}}^{\#}(\gamma(t), z)
$$

This last measure is the probability measure of annulus $S L E_{\kappa}$ as described in the previous section. 
Let us consider some implications of (16). For ease let us choose $D=\mathbb{D}$ and let $T$ equal a constant $-t$. We will consider what happens as $t \rightarrow \infty$. In this case the typical radius of $\gamma_{t}$ is of order $e^{-t}$. On $\mathbb{D}, F_{T}(z)$ is about $e^{t} z$ and $D_{T}$ is very close to the annulus $\mathcal{A}^{t}=\left\{1<|z|<e^{t}\right\}$. Hence $\left|F_{T}^{\prime}(z)\right|^{b} \sim e^{b t}$. Since $\Lambda^{*}\left(\gamma_{T}, \partial \mathbb{D}\right) \sim-\log \log e^{t}$, we get

$$
\exp \left\{\frac{\mathbf{c}}{2} \Lambda^{*}\left(\gamma_{T}, \partial D\right)\right\} \asymp t^{-\mathbf{c} / 2}
$$

We also have $\mu_{C}\left(\gamma_{T}\right)=e^{\tilde{b} t}$.

It is our hope that $\mu_{D}(0, z)$ is the reversal of $\mu_{D}(z, 0)$ and hence we would expect it to have the same total mass which in this case is $H_{\mathbb{D}}(1,0)$. We also expect this to be the total mass on the measure of paths $\gamma_{t}$, so this gives

$$
c_{0} e^{\tilde{b} t} t^{-\mathbf{c} / 2} e^{b t} H_{\mathcal{A}^{t}}\left(1, e^{t}\right) \sim H_{\mathbb{D}}(1,0), \quad t \rightarrow \infty
$$

(For large $t$, we expect that $H_{\mathcal{A}^{t}}\left(e^{i \theta}, e^{t+i \theta_{1}}\right) \sim H_{\mathcal{A}^{t}}\left(1, e^{t}\right)$.) This gives the conjecture

$$
H_{\mathcal{A}^{t}}\left(1, e^{t}\right) \sim c_{1} e^{-(b+\tilde{b}) t} t^{\mathbf{c} / 2}
$$

If we dilate by a factor of $e^{-t}$, and let $r=e^{-t}$, this gives the prediction

$$
H_{\mathcal{A}_{r}}(r, 1) \sim c_{1} r^{\tilde{b}-b}[\log (1 / r)]^{\mathbf{c} / 2} .
$$

If $\kappa=2$, we know that

$$
H_{\mathcal{A}_{r}}(r, 1)=h_{\mathcal{A}_{r}}(r, 1) \sim \frac{c}{r \log (1 / r)},
$$

which verifies the prediction in this case.

\section{The Brownian loop measure}

There are a number of equivalent ways to define the (Brownian) loop measure. While it is a measure on unrooted loops, it is easiest to define it in terms of rooted loops and then "forget the roots". One defines the measure on $\mathbb{C}$ and then defines it on subdomains by restriction.

The usual way is by considering the measure on rooted loops given by

$$
\frac{1}{2 \pi t^{2}} d t \times \text { area } \times \text { Brownian bridge. }
$$

Here "Brownian bridge" refers to the probability measure on paths of time duration 1 given by Brownian motion conditioned to be at the origin at time 1 . This is what one gets if one considers choosing the root uniformly over an unrooted loop. While this is a good way to define it for showing conformal invariance, there are equivalent definitions that are easier for computation. 
One way is to associate with each unrooted loop a rooted loop whose root is the point of smallest imaginary part. By doing this we write the measure as

$$
\frac{1}{2 \pi t^{2}} d t \times \text { area } \times \text { Brownian bridge/ bubble. }
$$

Here Brownian bridge/bubble refers to the probability measure on Brownian paths of time duration 1 conditioned to return to the origin at time 1 and stay in the upper half plane between times 0 and 1. A Brownian bridge/bubble consists of two independent one-dimensional processes: a Brownian bridge in the $x$-component and a Brownian excursion in the $y$ component. The state space for this measure are triples $\left(t, z, \gamma^{*}\right)$ where $\gamma^{*}$ is a loop in the upper half plane of time duration one beginning and ending at the origin. We associate to this triple the loop $\gamma$ which is obtained from $\gamma^{*}$ by Brownian scaling so that it has time duration $t$ and then translated so that it is rooted at $z$. The expression (17) gives a measure on loops rooted at their point of minimal imaginary part and the Brownian loop measure is obtained by "forgetting the root".

This leads to studying Brownian boundary bubbles. The Brownian boundary bubble measure in $\mathbb{H}$ at $0, m_{\mathbb{H}}(0)$, is an infinite measure on loops (or bubbles) rooted at 0 and otherwise staying in $\mathbb{H}$. Let $\xi^{\#}$ denote the probability measure associated to Brownian bridge/bubbles of time duration one and let $\xi_{t}^{\#}$ denote the similar probability measure for time duration $t$ obtained by usual Brownian scaling. Then we define

$$
m_{\mathbb{H}}(0)=\int_{0}^{\infty} \frac{1}{2 t^{2}} \xi_{t}^{\#} d t .
$$

Note that a factor of $\pi$ has been included. We can write the Brownian loop measure as

$$
\frac{1}{\pi} \int_{-\infty}^{\infty} \int_{-\infty}^{\infty} m_{\mathbb{H}+i y}(x+i y) d x d y
$$

where $m_{\mathbb{H}+i y}(x+i y)$ is defined in the obvious way by translation of $m_{\mathbb{H}}(0)$.

Another way to obtain the bubble measure is as follows. If $z \in \mathbb{H}, x \in \mathbb{R}$, let $\hat{m}_{\mathbb{H}}(z, x)$ denote the measure on paths corresponding to Brownian motion starting at $z$ and leaving $\mathbb{H}$ at $x$. It is normalized to have total measure equal to the Poisson kernel which we recall is $h_{D}(z, x) / \pi$. We then define

$$
m_{\mathbb{H}}(0)=\pi \lim _{\epsilon \rightarrow 0+} \epsilon^{-1} \hat{m}_{\mathbb{H}}(i \epsilon, 0) .
$$

To see this (and to check the multiplicative constant), it is easiest to split the loop into independent imaginary and real parts. If we start a one-dimensional Brownian motion at $\epsilon>0$ then the time of the first visit to the origin has density

$$
\frac{\epsilon}{\sqrt{2 \pi t^{3}}} e^{-\epsilon^{2} /(2 t)}
$$

If we divide by $\epsilon$ let $\epsilon$ go to zero we see that the the density for one-dimensional loops of time duration $t$ restricted to stay positive is given by $\left(2 \pi t^{3}\right)^{-1 / 2}$. The real part is a Brownian 
loop with no restrictions; it has density $(2 \pi t)^{-1 / 2}$. Hence the two-dimensional $\mathbb{H}$-bubbles have density $\left(2 \pi t^{2}\right)^{-1}$ which agrees with (18) up to the factor of $1 / \pi$.

For each subdomain $D \subset \mathbb{H}$ with $\operatorname{dist}(0, \mathbb{H} \backslash D)>0$, we define $\Gamma(0, D)=\Gamma(0, D ; \mathbb{H})$ to be the $m_{\mathbb{H}}(0)$ measure of the set of loops that intersect $\mathbb{H} \backslash D$. Although $m_{\mathbb{H}}(0)$ is an infinite measure, $\Gamma(0, D)$ is finite. The bubble measure is normalized so that $\Gamma\left(0, \mathbb{D}^{+}\right)=1$ where $\mathbb{D}^{+}=\mathbb{H} \cap \mathbb{D}$, see $(21)$. The Brownian bubble measure $m_{D}(z)$ can be defined similarly to (20) for general domains $D$ and smooth boundary points $z$. It is conformally covariant: if $f: D \rightarrow f(D)$ is a conformal transformation, and $z, f(z)$ are smooth boundary points of $D, f(D)$ respectively, then

$$
f \circ m_{D}(z)=\left|f^{\prime}(z)\right|^{2} m_{f(D)}(f(z))
$$

The Brownian bubble measure also satisfies the restriction property: if $D \subset D_{1}, z$ is a smooth boundary point of $D$ and $\partial D, \partial D_{1}$ agree in neighborhoods of $z$, then $m_{D}(z)$ is $m_{D_{1}}(z)$ restricted to loops in $D$. From (20), we get the formula

$$
\Gamma(0 ; D)=\int_{\partial D \cap \mathbb{H}} h_{\mathbb{H}}(z, 0) d \hat{m}_{D}(0, z)
$$

where $\hat{m}$ denotes the excursion measure. (Recall that we have defined $h_{D}$ to be $\pi$ times the usual Poisson kernel.) If $\partial D \cap \mathbb{H}$ is smooth, we can write

$$
\Gamma(0 ; D)=\frac{1}{\pi} \int_{\partial D \cap \mathbb{H}} h_{\mathbb{H}}(z, 0) h_{\partial D}(0, z)|d z| .
$$

In the example $D=\mathbb{D}^{+}, h_{\mathbb{H}}\left(e^{i \theta}, 0\right)=\sin \theta$, and by choosing a suitable conformal transformation, one can check that $h_{\mathbb{D}^{+}}\left(0, e^{i \theta}\right)=2 \sin \theta$ and hence

$$
\Gamma\left(0, \mathbb{D}^{+}\right)=\frac{1}{\pi} \int_{0}^{\pi}(\sin \theta)(2 \sin \theta) d \theta=1 .
$$

If $D_{1} \subset D$, we define $\Gamma\left(z, D_{1} ; D\right)$ to be the $m_{D}(z)$ measure of loops that intersect $D \backslash D_{1}$. If $f: D \rightarrow f(D)$ is a conformal transformation, conformal covariance implies

$$
\Gamma\left(z, D_{1} ; D\right)=\left|f^{\prime}(z)\right|^{2} \Gamma\left(f(z), f\left(D_{1}\right) ; f(D)\right)
$$

(assuming smoothness at the boundary).

The expression (19) uses a "foliation" of $\mathbb{C}$ by horizontal lines. By conformal covariance, we can use other foliations, say by concentric circles, which gives the expression

$$
\frac{1}{\pi} \int_{0}^{2 \pi} \int_{0}^{\infty} m_{r \mathbb{D}}\left(r e^{i \theta}\right) r d r d \theta
$$

This formulation chooses the root of the loop to be the point of greatest absolute value. By inversion, we could also choose the point of smallest absolute value giving the expression

$$
\frac{1}{\pi} \int_{0}^{2 \pi} \int_{0}^{\infty} m_{\mathbb{C} \backslash r \overline{\mathbb{D}}}\left(r e^{i \theta}\right) r d r d \theta .
$$


Note that we are using the fact that the scaling exponent for the Brownian bubble measure is 2 and hence the derivative term does not appear in area integrals.

We show how to determine asymptotics of some quantities related to the loop measure. Let $\mathbb{D}_{R}=\{|z|<R\}$ and $\mathbb{D}_{R}^{+}=\mathbb{D}_{R} \cap \mathbb{H}$. We start by showing that

$$
\Lambda\left(\mathbb{D}, \partial \mathbb{D}_{R} ; \mathbb{D}_{2 R}\right) \sim \frac{\log 2}{\log R}, \quad R \rightarrow \infty .
$$

Using (23), we get

$$
\Lambda\left(\mathbb{D}, \partial \mathbb{D}_{R} ; \mathbb{D}_{2 R}\right)=\frac{1}{\pi} \int_{0}^{2 \pi} \int_{0}^{1} \Gamma\left(r e^{i \theta}, \mathbb{D}_{R} \backslash \overline{\mathbb{D}_{r}} ; \mathbb{D}_{2 R} \backslash \overline{\mathbb{D}_{r}}\right) r d r d \theta
$$

If $R>1$, the probability that a Brownian motion starting at $R e^{i \theta}$ hits the circle of radius 1 before hitting the circle of radius $2 R$ is

$$
\frac{\log 2}{\log (2 R)}
$$

Hence as $R \rightarrow \infty$,

$$
h_{\mathbb{D}_{2 R} \backslash \mathbb{D}}\left(R e^{i \theta}, e^{i \phi}\right) \sim \frac{1}{2} \frac{\log 2}{\log R} .
$$

(we use the fact that in the $R \rightarrow \infty$ limit, the left-hand side does not depend on the angles $\theta, \phi)$. If $f(z)=f_{R}(z)$ denotes the probability that a Brownian motion starting at $z$ reaches the circle of radius $R$ before the circle of radius 1 then

$$
f(z)=\frac{\log |z|}{\log R}, \quad 1 \leq|z| \leq R .
$$

In particular, its normal derivative at $e^{i \phi}$ equals $1 / \log R$. Therefore,

$$
\Gamma\left(e^{i \theta}, \mathbb{D}_{R} \backslash \overline{\mathbb{D}} ; \mathbb{D}_{2 R} \backslash \overline{\mathbb{D}}\right) \sim \frac{\log 2}{2 \log ^{2} R}, \quad R \rightarrow \infty
$$

By conformal covariance, we get

$$
\Gamma\left(r e^{i \theta}, \mathbb{D}_{R} \backslash \overline{\mathbb{D}_{r}} ; \mathbb{D}_{2 R} \backslash \overline{\mathbb{D}_{r}}\right)=r^{-2} \Gamma\left(e^{i \theta}, \mathbb{D}_{R / r} \backslash \overline{\mathbb{D}} ; \mathbb{D}_{2 R / r} \backslash \overline{\mathbb{D}}\right) \sim \frac{\log 2}{2 r^{2} \log ^{2}(R / r)}
$$

Therefore,

$$
\frac{1}{\pi} \int_{0}^{2 \pi} \int_{0}^{1} \Gamma\left(r e^{i \theta} ; \mathbb{D}_{R} \backslash \overline{\mathbb{D}_{r}} ; \mathbb{D}_{2 R} \backslash \overline{\mathbb{D}_{r}}\right) r d r d \theta \sim \frac{1}{\pi} \int_{0}^{2 \pi} \int_{0}^{1} \frac{\log 2}{2 r^{2} \log ^{2}(R / r)} r d r d \theta=\frac{\log 2}{\log R} .
$$

We will now show how to derive

$$
\Lambda\left(\mathbb{D}_{1 / R}^{+}, \partial \mathbb{D}^{+} ; \mathbb{H}\right)=\Lambda\left(\mathbb{D}^{+}, \partial \mathbb{D}_{R}^{+} ; \mathbb{H}\right) \sim R^{-2}, \quad R \rightarrow \infty
$$


The first equality holds by conformal invariance. Using (22) and the restriction property, we have

$$
\Lambda\left(\mathbb{D}^{+}, \partial \mathbb{D}_{R}^{+} ; \mathbb{H}\right)=\frac{1}{\pi} \int_{0}^{\pi} \int_{0}^{1} \Gamma\left(r e^{i \theta}, \mathbb{D}_{R}^{+} \backslash \overline{\mathbb{D}_{r}} ; \mathbb{H} \backslash \overline{\mathbb{D}}_{r}\right) r d r d \theta
$$

By conformal covariance,

$$
\Gamma\left(r e^{i \theta}, \mathbb{D}_{R} \backslash \overline{\mathbb{D}_{r}^{+}} ; \mathbb{H} \backslash \overline{\mathbb{D}}_{r}\right)=r^{-2} \Gamma\left(e^{i \theta}, \mathbb{D}_{R / r} \backslash \overline{\mathbb{D}} ; \mathbb{H} \backslash \overline{\mathbb{D}}\right)
$$

The map $g(z)=z+z^{-1}$ maps $\mathbb{H} \backslash \mathbb{D}$ conformally onto $\mathbb{H}$ with $\left|g^{\prime}\left(e^{i \theta}\right)\right|^{2}=4 \sin ^{2} \theta$. As $R \rightarrow \infty$,

$$
\begin{aligned}
\Gamma\left(e^{i \theta}, \mathbb{D}_{R / r} \backslash \overline{\mathbb{D}} ; \mathbb{H} \backslash \overline{\mathbb{D}}\right) & =\left|g^{\prime}\left(e^{i \theta}\right)\right|^{2} \Gamma\left(g\left(e^{i \theta}\right), g\left(\mathbb{D}_{R / r} \backslash \overline{\mathbb{D}}\right)\right) \\
& \sim\left|g^{\prime}\left(e^{i \theta}\right)\right|^{2} \Gamma\left(0, \mathbb{D}_{R / r}\right) \\
& =4 \sin ^{2} \theta(r / R)^{2}
\end{aligned}
$$

Since

$$
\frac{1}{\pi} \int_{0}^{\pi} \int_{0}^{1} 4 r \sin ^{2} \theta d r d \theta=1
$$

we get (25) by plugging into (26).

The set $\overline{\mathbb{D}_{\sqrt{r}}^{+}}$has half-plane capacity $r$. One can show that if $K_{r}$ is any collections of sets decreasing to 0 as $r \rightarrow 0+$, with half-plane capacity $r$, then

$$
\Lambda\left(K_{r}, \mathbb{H} \backslash \mathbb{D} ; \mathbb{H}\right) \sim r
$$

and more generally

$$
\Lambda\left(K_{r}, \mathbb{H} \backslash D ; \mathbb{H}\right) \sim r \Gamma(0, D) .
$$

A straightforward calculation also gives for simply connected $D, \Gamma(0, D)=-S f(0) / 6$ where $f: D \rightarrow \mathbb{H}$ is a conformal transformation. Therefore, if $\gamma$ is a simple curve parametrized so that $\gamma_{t}$ has half-plane capacity at,

$$
\Lambda\left(\gamma_{t}, \mathbb{H} \backslash D ; \mathbb{H}\right)=a \int_{0}^{t} \Gamma\left(U_{s} ; g_{s}(D)\right) d s .
$$

This holds for all $D$ whether simply connected or not. For simply connected $D$ we also get

$$
\Lambda\left(\gamma_{t}, \mathbb{H} \backslash D ; \mathbb{H}\right)=-\frac{a}{6} \int_{0}^{t} S \Phi_{g_{s}(D)}\left(U_{s}\right) d s
$$

\subsection{Annulus}

Let $\mathcal{A}_{s, r}$ denote the annulus $\mathcal{A}_{s, r}=\{s<|z|<r\}$ and $\mathcal{A}_{s}=\mathcal{A}_{s, 1}$. The loop measure in the annulus $\mathcal{A}_{s}$ can be written using (22) as

$$
\frac{1}{\pi} \int_{0}^{2 \pi} \int_{s}^{1} m_{s, r}\left(r e^{i \theta}\right) r d r d \theta
$$


where $m_{s, r}=m_{\mathcal{A}_{s, r}}$. We can write

$$
m_{s, r}(z)=\sum_{k=-\infty}^{\infty} m_{s, r, k}(z)
$$

where $m_{s, r, k}(z)$ denotes $m_{s, r}(z)$ restricted to loops with winding number $k$ about the origin. This is a finite measure if $k \neq 0$; let us denote the total mass by $J(s, r, k)$. By conformal covariance,

$$
J(s, r, k)=r^{-2} J(s / r, 1, k) .
$$

Let $F(z)=e^{i z}$. For $k \neq 0$,

$$
m_{s, 1, k}(1)=\pi F \circ \hat{m}_{D_{t}}(0,2 \pi i k)
$$

where $\hat{m}$ denotes the excursion measure, $t=-\log s$ and $D_{t}=\{x+i y: 0<y<t\}$. Also,

$$
m_{s, 1,0}(1)=F \circ m_{D_{t}}(0)
$$

(The excursion measure does not have the factor of $\pi$ in its definition but the bubble measure does. This is why these expressions differ by that factor.)

Let $f_{t}(z)=e^{\pi z / t}$. Then $f_{t}$ maps $D_{t}$ conformally onto $\mathbb{H}$ and hence

$$
\begin{aligned}
h_{D_{t}}(0,2 \pi k) & =\left|f_{t}^{\prime}(0)\right|\left|f_{t}^{\prime}(2 \pi k)\right| h_{\mathbb{H}}\left(f_{t}(0), f_{t}(2 \pi k)\right) \\
& =(\pi / t)^{2} e^{2 k \pi^{2} / t}\left[e^{2 k \pi^{2} / t}-1\right]^{-2} \\
& =\frac{\pi^{2}}{4 t^{2} \sinh ^{2}\left(k \pi^{2} / t\right)} \\
& =\frac{1}{4 \pi^{2} k^{2}}\left[1+O\left(k^{2} / t^{2}\right)\right]
\end{aligned}
$$

For fixed $k$,

$$
J(s, r, k) \sim r^{-2} \frac{1}{4 \pi^{2} k^{2}} .
$$

Hence (using the error estimate above, we omit the details)

$$
\frac{1}{\pi} \int_{0}^{2 \pi} \int_{s}^{1} J(s, r, k) r d r d \theta \sim \frac{1}{2 \pi^{2} k^{2}} \log (1 / s) .
$$

Using $\sum_{k \neq 0} k^{-2}=\pi^{2} / 3$, we see that the measure of the set of loops with nonzero winding number is asymptotic to $(1 / 6) \log (1 / s)$.

\section{References}

[1] A. Belavin, A. Polyakov, A. Zamolodchikov (1984), Infinite conformal symmetry of critical fluctuations in two dimensions, J. Stat. Phys. 34, 763-774. 
[2] A. Belavin, A. Polyakov, A. Zamolodchikov (1984), Infinite conformal symmetry in two-dimensional quantum field theory. Nuclear Phys. B 241, 333-380.

[3] M. Kozdron, G. Lawler (2007). The configurational measure on mutually avoiding SLE paths, in Universality and Renormalization: From Stochastic Evolution to Renormalization of Quantum Fields, I. Binder, D, Kreimer, ed., Amer. Math. Soc., 199-224.

[4] G. Lawler (2005). Conformally Invariant Processes in the Plane, American Mathematical Society.

[5] G. Lawler (2006), The Laplacian- $b$ random walk and the Schramm-Loewner evolution, Illinois J. Math. 50, no. 1-4, 701-746.

[6] G. Lawler (2009), Schramm-Loewner evolution, notes from lectures at 2008 IAS-Park City Institute, to appear.

[7] G. Lawler, O. Schramm, W. Werner (2003), Conformal restriction: the chordal case, J. Amer. Math. Soc. 16, 917-955.

[8] G. Lawler, W. Werner (2004), The Brownian loop soup, Probab. Theory Related Fields 128, 565-588.

[9] O. Schramm (2000), Scaling limits of loop-erased random walks and uniform spanning trees, Israel J. Math. 118, 221-288.

[10] W. Werner (2004), Random planar curves and Schramm-Loewner evolutions, Ecole d'Eté de Probabilités de Saint-Flour XXXII - 2002, Lecture Notes in Mathematics 1840, Springer-Verlag, 113-195.

[11] W. Werner (2008), The conformally invariant measure on self-avoiding loops, J. Amer. Math. Soc. 21, 137-169.

[12] D. Zhan (2008). Reversibility of chordal SLE, to appear in Annals Probab. 